\title{
CYCLICAL AND SECULAR VARIATIONS \\ OF SOLAR ACTIVITY
}

\author{
G. V. KUKLIN \\ Sibizmiran, Irkutsk, U.S.S.R.
}

It is difficult to prepare an invited paper on topics which are $\mathrm{ABC}$ for any specialist in Solar Physics. I considered my task as a systematization of a lot of information about cyclic and secular variations of solar activity rather than an account of new striking results in order to illustrate a certain concept. I beg your pardon in advance for possible defects in my report and for lack of time to quote all related papers.

\section{Philosophy}

\subsection{ASPECTS, CONCEPTS, INDICES}

One can speak about three aspects of the study of solar activity, as a whole, although usually no essential difference is made between them. The frequency-time aspect of solar activity study involves the consideration of a set of time variations in different solar activity indices and the determination of the power spectrum of quasiharmonic and cyclic variations. The spatial aspect implies the study of the spatial distribution of solar activity phenomena and, in particular, the clearing up of the degree of homogeneity or inhomogeneity in the distribution of various solar activity indices in latitude and longitude. The study of the significance of individual phenomena, which depends directly on the phenomena lifetime, reflects the importance of these investigations.

The basic concept crystallized from results of papers done in the past $20 \mathrm{yr}$, is associated with the division of various solar activity phenomena and indices characterizing the former into two large classes. This idea was proposed for the first time by Kopecký (1958). To the first class belong indices which predominantly reflect how frequently new formations appear on the Sun. A nearly uniform distribution in longitude and a relatively low concentration in latitude is typical for them. The main time variation is the 11-year cycle. Time variations in the spatial distribution pattern are influenced by the differential rotation. The indices pertaining to the second class contain information on phenomena importance. Their spatial distribution corresponds to a substantial inhomogeneity in longitude (the presence of so-called active longitudes), and the concentration in the low latitude region below $20^{\circ}$. Time variations of these indices mainly are represented by a secular $(80-90 \mathrm{yr})$ cycle. It is remarkable that the spatial distribution of indices is not influenced by the differential rotation, but it rotates rigidly with a constant angular velocity. The presence of the two classes requires a different approach to indices which contain frequency and importance aspects of the phenomena. The mixing of these factors, as a rule, leads to uncertainty and sometimes even to contradictions in the results, depending strongly on data processing. 
All the wealth and diversity of the forms of solar activity phenomena are coded in a compact numerical form by various indices. These indices can be classified in different ways. First, we can subdivide them into basic physical indices and derived indices. To the former pertain numerical characteristics which are observed and measured directly: sunspot number, sunspot coordinates, sunspot area, flare brightness, radio emission level, and so forth. The latter imply all indices resulting from some processing or other of basic indices or their combination: Wolf numbers, flare indices, mean characteristics, fluctuation indices, asymmetry indices, and so on. Secondly, indices are subdivided into static and dynamic ones. Static indices, to which the majority of values pertain, are obtained by either averaging procedure, contain information about the level of the measured value. Dynamic indices determine the variability of either values. These are various measures of fluctuations, derivative evaluations, and similar constructions. Probably, essentially more emphasis than has been made so far, should be placed on dynamic indices due to their greater efficiency.

As an example let us consider a certain dynamic index representing the relative speed of variation $[(1 / C)(\mathrm{d} C / \mathrm{d} t)]$ of a value $C$. The values of this index correspond to the inverse value of the characteristic-time variation of the initial parameter. Usually objects being studied represent a complicated open system with many couplings. One of the tasks of the functioning of such a system is the adaptation to variations of external and internal conditions. This process is characterized by a definite delay time $\tau$. Until variations in the system occur during a time greater than $\tau$, necessary recombinations take place without essential qualitative changes in the actual system. In a case where absolute values of the dynamic index are so great that the corresponding times are less than $\tau$, disagreement takes place in the system which can qualitatively affect this system. For that reason, dynamic indices can prove to be highly effective for forecasting.

As mentioned above, indices can be divided into importance and frequency indices. Kopecký $(1958,1967)$ proposed the frequency of new formation phenomena on the Sun $f_{0}$ and their mean life-time $T_{0}$ to be considered as primary indices. Originally, these values were determined by him for sunspots. In this case the formation distribution density in respect to life-time $F(T)$ must play an essential role. For the case when these characteristics are time independent, corresponding mathematical methods were developed which made it possible to express the indices usually being used, with the help of the primary $f_{0}$ and $T_{0}$ :

$$
\begin{array}{ll}
\text { observed sunspot number } & N=f_{0} T_{0}, \\
\text { Wolf number } & W=K f_{0} T_{0}, \\
\text { sunspot mean area } & \bar{S}=C f_{0} T_{0}^{k}, \quad k=1 \text { or } 2 .
\end{array}
$$

Primary indices by themselves cannot be determined directly from observations because they are produced values. The methods to compute them are described by Kopecký $(1958,1967)$. In recent years Kopecký developed mathematical methods for the case when primary indices and required distribution functions are time dependent $(1972,1973,1974,1975)$. Naturally one expects that indices for which determination and computation areas, life-time, intensities were used will predomin- 
antly reflect the behaviour of importance indices; moreover, the frequency index effects can also reveal themselves, although to a lesser extent. Indices describing the number of observed formations, to a greater extent are similar frequency indices. Unfortunately, practically in all cases, the observed values cannot be reduced to primary indices because the corresponding expressions contain coefficients in an unknown manner depending on time and coordinates. Only by averaging for time intervals of order $1 \mathrm{yr}$ and over coordinate intervals of several tens of degrees, can one attempt to make such reduction and in this case the statistical errors of the primary indices obtained, turn out rather large, so that an additional smoothing of the results is required.

Finally, indices used in various investigations can be divided into standard and non-standard ones. The former involve widely used indices determined according to standard programs and published in various issues as catalogues, Bulletin 'Solar Data' etc. Usually they do represent the basic material of the studies being carried out. Non-standard indices introduced in individual papers, are not broadly accessible and are not available in the form of long homogeneous series.

\subsection{The frequency spectrum of variations}

\subsection{THE FREQUENCY SPECTRUM OF VARIATIONS}

According to the approach accepted now, we shall take frequency spectra of the index time series as a basis of classification of solar activity variations. There seems to be no particular need to list a great number of papers in which either periods were chosen using different methods. A summary of these results, being far from complete, is given in Table I.

The overwhelming majority of authors have used Wolf number series as initial material, an index in common use, convenient due to the presence of relatively long series, but in a certain degree inappropriate because of its complicated nature. The Wolf number series of monthly values cover now more than $220 \mathrm{yr}$; for 11 -year cycle minimum or maximum epochs these are followed back to 1610 . The investigation of considerably longer periods is carried out using materials about phenomena which to some extent are associated with solar activity. This includes tree ring thickness measurements, deposit accumulation, chronicles in which evidences are noted on outstanding natural phenomena: drought, aurorae, bright comets, etc. On the basis of such materials one can roughly reconstruct the run of solar activity in a qualitative or even semi-quantitative scale, permitting an approximate reduction to Wolf numbers (Schove, 1955).

Data on sunspot group importance cover only $100 \mathrm{yr}$ of regular observations. Evidences on sunspot flocculi, prominences, filaments, magnetic fields can be utilized practically since the beginning of the 20th century. Finally, fairly detailed and reliable evidence on flares, corona and solar radio emission are available from the beginning of the IGY, i.e., for about 2 cycles, although there are less reliable data which are accessible starting with the post-war years.

It is advisable to speak about three classes of periods which have been found: (a) periods of tens and hundreds of years; (b) periods within 7-25 yr; (c) periods less than 


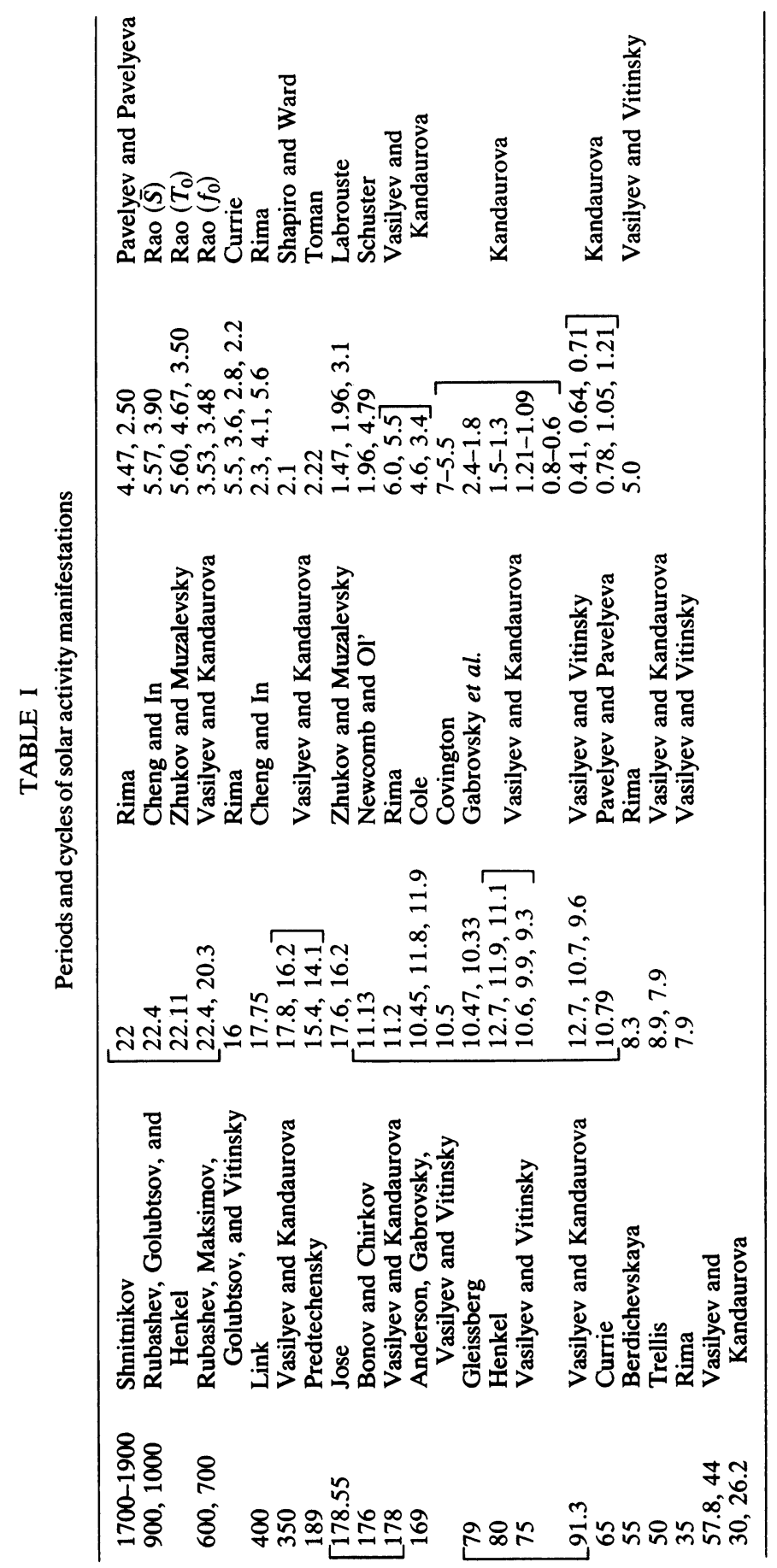


$6 \mathrm{yr}$. The class is composed of supersecular and secular cycles, as well as a number of intermediate values of periods. Recurrence periods of various geophysical phenomena correspond to a great many of these values. The second class is very rich with spectral lines, of which the 22-year and 11-year cycles are the main. The frequency spectrum structure in this region is complicated and variable since many periodicities are not always present and are able to disappear during some time intervals. Otherwise, the spectral lines have finite width independent of the method by which they are detected. A greater variety of periods is observed in the third class. A special analysis by Kandaurova (1971) distinguished five subclasses of short periods, fairly clear-cut in time of Wolf numbers (roughly, frequency index) as well as of the sunspot area (greater contribution of the importance index). The last subclass with periods less than one year essentially exhibits effects of fluctuation of separate indices which, as considered now by most investigators, are caused by the existence of large individual active regions. It is typical that short periods correspond to non-stationary variations lasting about one-three 11 -year cycles. It should be noted that some periodic components appear simultaneously, their periods being in whole number ratio between each other as well as with regard to the 11-year cycle, which qualified them to be considered as overtones of the basic 11-year cycle.

We have already mentioned that indices characterizing the phenomena importance manifest themselves most strikingly in the secular cycle, and for characteristics of new formation origin the 11-year cyclic periodicity is more typical. This statement is not absolute in the sense that a secular variation in $f_{0}$ and a 11-year variation in $T_{0}$ can be found. Kopecký and Kuklin (1971) showed the presence of an 11-year variation in $T_{0}$ and proposed to explain secondary 11-year cycle maxima, found by Gnevyshev (1963), through this very variation. Once one looks at the diagram of $f_{0}$ values, the 22-year component is found at once, but it would be irresponsible to speak about the presence of a secular cycle in $f_{0}$ using a series of such short length. Really, the autospectra of $f_{0}$ and $T_{0}$ contain significant components with a period of about $11 \mathrm{yr}$. The component with $\sim 22$-yr period is present in the spectrum of $f_{0}$, but it is negligible in the spectrum of $T_{0}$. Finally, the difference mentioned in secular variations is quite sharply illustrated by the diagrams of the cross spectrum modulus

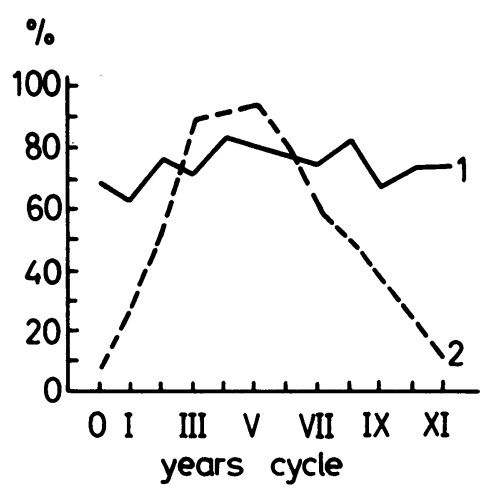

Fig. 1. The variation of primary indices $T_{0}(1)$ and $f_{0}(2)$ during the 11 -year cycle. 

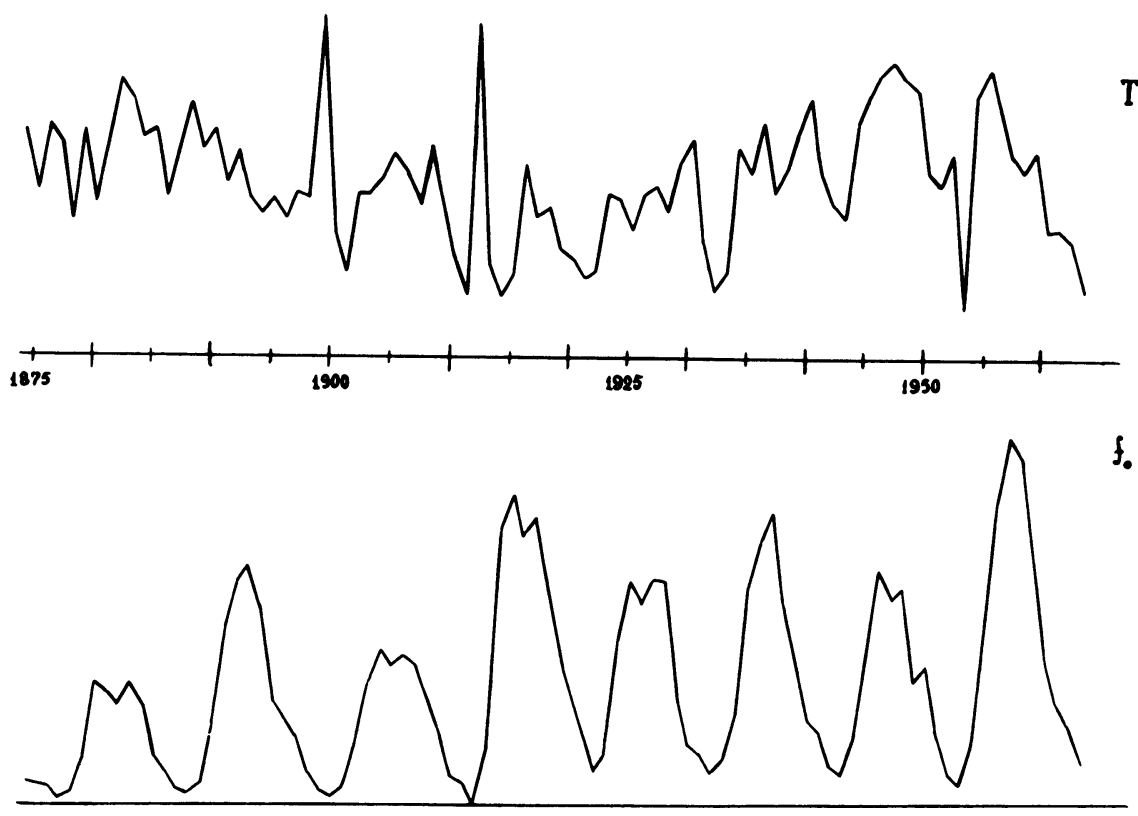

Fig. 2. The time-series of $f_{0}$ and $T_{0}$.
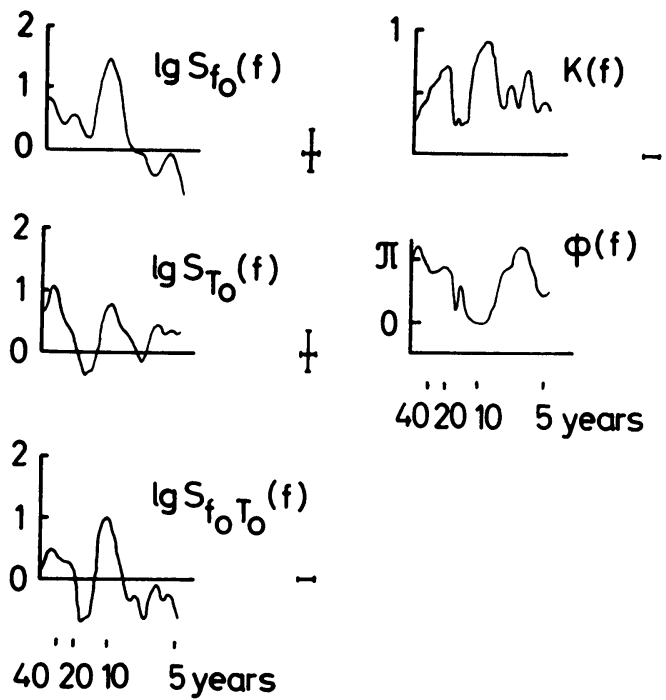

Fig. 3. The autospectra of $f_{0}$ and $T_{0}$, for the cross-spectrum, the coherence index $K$, the phase spectrum for the time-series of $f_{0}$ and $T_{0}$.

and coherence index. The diagram of spectral phases shows that the basic harmonics of 11-year variations of $f_{0}$ and $T_{0}$ are synphased, but the secondary harmonics are already nearly in counterphase which generally means that maxima of 11-year variations of $f_{0}$ and $T_{0}$ do not coincide. However, the coherence index for the 
secondary harmonic cannot be considered to be significant. This agrees quite well with the fact that the 11-year variation in $T_{0}$ varies in shape when transiting from the descending branch of the secular cycle to the ascending branch (Kopecký and Kuklin, 1971).

It should be noted that Romanchuk and Sergeeva (1974) detected an inverse dependence between $f_{0}$ and $T_{0}$, qualitatively invariable, but quantitatively distinct at different phases of the 11-year cycle. This regularity essentially reflects peculiarities of $f_{0}$ and $T_{0}$ spectra.

An analysis made by Vasilyev and Vitinsky (1969) has shown that sunspot areas, Wolf numbers and sunspot weighted latitudes have close basic periodic components $(10.7,169,9.6,75,12.7 \mathrm{yr})$. The components with periods 5.0, 7.9 and $16.1 \mathrm{yr}$ present in the Wolf number spectrum, do not appear always in spectra of the indices to a greater degree.

There are curious attempts made by some authors following the idea by Anderson (1939), to convert the normal series of Wolf numbers into a sign-changing series, ascribing, e.g. to Wolf numbers minus in odd cycles on the basis of the Hale law. In this case the spectrum is strongly simplified and only three components with periods 22.1, 17.6 and $16.2 \mathrm{yr}$ are left in it, which should be considered as 'basic' ones (Muzalevsky and Zhukov, 1968a, b). All variety of periods can be obtained as a result of the 'basic' oscillation interference and the assumption that the observed indices are the response of the Sun which is interpreted as a square detector. The invalidity of such an approach from the physical viewpoint and the absence of any gain in accuracy in the formal-mathematical sense were demonstrated by Vasilyev and Vitinsky (1969).

In connection with above, it is necessary to comment upon the problem of beats. The question is that many authors employing spectral or periodogram methods, obtained several 'basic' periods, the superposition of pairs of which provided all other periods, including even supersecular ones. More frequently data on a single index, e.g. Wolf numbers, were used. Pavelyev and Pavelyeva (1965) obtained three 'basic' periods of $10.8,4.5$ and $2.5 \mathrm{yr}$. Combining these, one can obtain cycles of $22,44,36$ and $85 \mathrm{yr}$. Combining the periods of 22.2 and 17.7 yr a secular cycle can be obtained (Cheng and In, 1965; Muzalevsky and Zhukov, 1968c). Similar considerations were also given by Cole (1973), Cohen and Lintz (1974) and others. However, a doubt inevitably arises as to reality of 'fictitious' secular and other cycles. In fact, the problem must be turned around. The secular cycle is a physical reality, but it has a length oscillating near a mean value of $80 \mathrm{yr}$. The result of its superposition with other physically real cycles (in simplest case, the amplitude modulation) does produce a 'fictitious' non-stationary fine structure of frequency spectra of many non-primary indices.

\subsection{Mathematical MODEls}

Recently it has become popular to construct mathematical models of solar periodicity without going deeply into the physical nature. For example, it is postulated that the series of Wolf numbers can be considered as a random process, and characteristics of such process are analyzed. The construction of mathematical models implies the application of the apparatus of random process theory, but the known series of 
indices can be approximated neither by a determinated process nor by a stochastic one in the pure form. According to estimations by Vasilyev and Vitinsky (1969) about $90 \%$ of the process dispersion falls on the side of regular variations of solar activity, and the rest on the side of the random component. For this reason an analysis of the nature of each index oscillation by statistical methods must precede the construction of mathematical models.

According to Drozdov (1950) all oscillations of either index may be classified as follows: (1) random oscillations, (2) disturbances, (3) rhythms, (4) periodic oscillations, (5) latent periodicity (or periodicity with disturbances). Random oscillations can be rather well presented with the help of a model of quasi-white noise. Periodic oscillations have practically constant period, an amplitude and a phase. Rhythmic oscillations represent an interchange of disturbances (aperiodic deviations) of distinct signs with certain mean period and with an accumulation of phase difference, occurring in a gradual or stepped manner. Latent periodicity represents oscillations with random varying periods, amplitudes and phases near their mean values. In contrast to rhythms, no accumulation of phase difference takes place here. Investigations by Newcomb (1901) and Ol' (1960) have shown that 11-year cycles in these terms are an example of latent periodicity with a mean period of 11-13 yr. Analogous statements can be made with respect to secular cycles, the mean duration of which according to Gleissberg (1955) is 7.111 -year cycles. However, deviations of observed epochs of 11-year cycle extrema from the calculated ones are not purely random: when smoothed over 4 cycles, deviations exhibit a regular interchange of deviation signs. This is an indication of the influence of a higher range cycle. Therefore, the totality of cycles of different lengths can represent some hierarchy (structural co-ordination). This idea was conceived by Eigenson (1963). Fluctuations of solar activity according to Vitinsky (1961b) must be referred to the random oscillation type, and even for this reason one can speak neither about a morphological likeness nor a hierarchic relation of fluctuations as an example of the 11-year cycle.

The superposition hypothesis was proposed by Wolf at the end of the last century, and it considers the curves of index variations of solar activity as a result of superposition of many periodic components. The formalism and unwarranted determination in earlier papers of this type have led to a striking failure of this approach. Recently, the superposition hypothesis has been revived on the basis of new achievements of mathematical statistics and application of computers. In the new variant, solar activity is considered as a polyperiodic process with a rather great random component. However, no physical interpretation is provided to the set of 'basic' frequencies, and the set itself is formed on the principle of the best approximation of observed series. Naturally, the meaning of the procedures utilized is lost in this case.

To counterbalance the superimposition hypothesis, Waldmeier (1935) proposed an 'eruptive' hypothesis according to which each 11-year cycle represents an independent explosion. It played a great role in the study of internal regularities of 11-year cycles but proved to be inconsistent in the sense that it does not take into account couplings of the statistical and physical character of the neighbouring 11-year cycles. 
In a number of papers by Gudzenko and his colleagues, a model of the 'black box without input' was developed. On the basis of the series of Wolf numbers a model was constructed of the auto-oscillation system with one degree of freedom synchronized by a weak periodic signal. On one hand this model served as an additional argument in favor of the 'eruptive' hypothesis, as the physical interpretation of this model corresponds to the consideration of the Sun as a relaxation generator. On the other hand, using such an approach, physical links between cycles in a pair (Hale Law) and the presence of a secular cycle are neglected. So, this model possessed no vital capacity either.

If we consider more particular models of the formal kind, we should quote the results by Jakimiec (1969) who has shown that sunspot areas index deviation from smoothed values may be described as a stationary multiplicative random process, as well as the model of 'auto-regression-integrated sliding average' proposed by Phadke and Wu (1974) for the series of Wolf numbers. In the last model the value of $10.83 \mathrm{yr}$ was obtained for the period. In both cases, mathematical models are formal and have no concrete physical interpretation.

Thus, mathematical models of solar cycle periodicity proposed hitherto predominantly bore formal character, were essentially functionally-adequate ones and were of practical interest only for the approximation of initial data series.

\subsection{THE PROBLEM OF EXTERNAL EFFECTS ON THE SUN}

The finite aim of solar studies is to build a common theory of solar activity. Up to now, one cannot name any theory which could explain and describe the whole complicated pattern of spatial-time variations of solar activity, all hierarchy of the cycles of solar activity, the presence of a system of active longitudes, and the difference between indices of frequency and importance phenomena. Nearly all theories are limited by the 11- or 22-year cycle and Spoerer's law. A large amount of data still awaits corresponding theoretical constructions.

All theories of solar activity conceived up to now may be divided into three groups: (a) causes and sources of solar activity lies within the Sun itself; (b) external effects serve as a regulator of processes on the Sun; (c) external effects are the causes and sources of solar activity. Theories of the first group were intensively developed during the 20th century; they prefer mechanisms with direct contribution of magnetic fields, and they will be discussed in detail at this Symposium. For this reason, we will permit ourselves to pay some attention to the state of theories of the second and third groups.

Mostly, external effects imply the gravitational influence of planets upon solar processes. Recently, considerable interest has revived in this direction. Unfortunately, investigations very often are reduced only to a search of formal and statistical links between indices of solar activity and planetary configurations. The idea of planetary conditionality of phenomena on the Sun is very old (it was suggested as far back as Wolf). Supporters of this idea existed practically always, although nobody went into the question of what is the cycle of their number variation.

The accordance between the frequency spectrum of index variations of solar activity and the periods of planetary configuration recurrence, as a rule, serve as a 
basis for research in this direction. Particular attention is paid to Jupiter as the largest body of the solar system and as a planet with a rotation period close to the 11-year cycle length.

Mostly one considers tidal disturbances and variations in the solar center position with respect to the mass center of the whole solar system (baricentric motion of the Sun) as external influences, according to estimations by Anderson (1954), the total tidal solar gravitation force ratio is of order $5 \times 10^{-12}$. Trellis (1966a) has concluded that the tide height on the Sun does not exceed $1 \mathrm{~mm}$, however, the mean daily work of tidal forces of about $10^{35} \mathrm{erg}$ is comparable with the energy released by the most important flares $\sim 10^{33} \mathrm{erg}(1966 \mathrm{c})$. Displacements of the solar mass center in the baricentric coordinate system may exceed $2 R_{\odot}$. It is supposed that such motions may serve as a trigger to produce disturbances in the unstable convective zone.

Let us list the results giving evidence in favor of planetary effects. Suda (1962) found in the Wolf number series periods and semi-periods of the Earth and Jupiter rotation by means of a periodogram analysis. The joint influence of Mercury, Venus, Earth and Jupiter also was studied (their mean relative contributions are $1.0: 2.2: 1.0: 2.3$, and the influence of other planets can be neglected). A basic period of $178 \mathrm{yr}$ was found which together with its harmonics must determine secular variations of solar activity. Maksimov and Smirnov (1967) found considerable correspondence between structures of frequency spectra of variations of solar indices and planetary tides. Jose (1965) found the period of $178.8 \mathrm{yr}$ in the baricentric motion of the Sun and connected it with the same period in the Wolf number series (double secular cycle). The analysis of the Sun's position considering its baricentric motion, its velocity, its acceleration and acceleration change ('jerk'), taking account of all planets, led to a period of $11.08 \mathrm{yr}$ (Wood and Wood, 1965). Bigg (1967) distinguished the variation of Wolf numbers with the period of Mercury. Dauvillier (1970) showed that the height of the 11-year cycles is proportional to the value of the Sun's displacement in the baricentric coordinate system. Takahashi $(1967,1968)$ found in variations of tidal forces a period of $22.4 \mathrm{yr}$ and in addition distinguished a period of $11 \mathrm{yr}$ with a $2-\mathrm{yr}$ phase shift and a $\sim 0.7$ amplitude. A correspondence of periods was detected by Kolomeets et al. (1974). Trellis (1966b) found that the sunspot area and the number of new sunspot groups linearly depends on the tide height. Ambrož (1971) when comparing maps of calcium flocculi and the vertical component of tidal force came to the conclusion that there was a coupling between these characteristics in active longitudes.

Not a few empirical correlations were used with the aim of forecasting on the basis of planetary configuration analysis. In a number of papers Romanchuk conceived and applied a composite algorithm of prediction of solar activity depending on the planetary location with due regard for special empirical functions of action. A relation between 11-year cycle maxima and the Jupiter-Saturn quadrature was found here. Nemeth (1966) constructed a more simple algorithm utilizing the time interval between Venus-Earth and Jupiter-Earth conjunctions. This value is minimal during maximum epochs of 11 -year cycles and exhibits a mean period of $2 \times 11.2 \mathrm{yr}$. Shuvalov (1970) has found that planetary clustering in longitude corresponds to maxima of solar activity and blurring, conversely, to minima. Various combinations of planets lead to 11-year and 100-year periods. According to Liese (1971) the 
maxima are associated with Jupiter-Saturn quadratures and the minima with positions when the difference in longitudes of these planets is equal to $45^{\circ}$ or $225^{\circ}$. Prokudina (1973a) has found that an activity increase in a given region of the Sun is associated with the crossing by this region of a direction determined by planetary configuration. Later (1973b), an attempt was made to explain these correlations through tidal forces and variations of the angular orbital moment of the Sun. Separate important events on the Sun were predicted using planetary configurations (Nelson, 1963; Blizard, 1965). Analyzing resonance correlations for the solar system (Molchanov, 1966) Kozelov (1972), has found a correspondence between secular variations of resonance longitudes and superlong cycles of solar activity. According to the same study, the Earth's position, when its longitude is close to the resonance one, corresponds to activity increases on the Sun.

TABLE II

The resonance relationships (Kozelov)

\begin{tabular}{lcl}
\hline Longitudes & Periods & Possible cycles \\
\hline$\lambda_{1}=62.28-1.84 T$ & $196^{\mathrm{a}}$ & \\
$\lambda_{2}=187.08-1.25 T$ & 288 & $T_{2}-T_{1}=92^{\mathrm{a}}$ \\
$\lambda_{3}=87.90-0.40 T$ & 901 & $T_{1}=196^{\mathrm{a}}$ \\
$\lambda_{4}=182.95-0.74 T$ & 487 & $T_{1}-T_{3}+T_{5}=178^{\mathrm{a}}$ \\
$\lambda_{5}=188.19-0.41 T$ & 883 & $T_{5}-T_{4}=396^{\mathrm{a}}$ \\
$\lambda_{6}=61.18-0.36 T$ & 1017 & $T_{3}=901^{\mathrm{a}}$ \\
$\lambda_{7}=75.28-0.08 T$ & 4270 & $T_{3}-T_{2}=613^{\mathrm{a}}$ \\
$\lambda_{8}=266.61-0.04 T$ & 9747 & \\
\hline
\end{tabular}

All the above correlations cause a mixed reaction. On the one hand they, to a certain extent, are convincing and stimulate further studies in this direction. On the other hand, there is no impressive physical explanation of the links found. Invalidity of all mechanisms in the energetic aspect was shown more than once (Ferris, 1969; Vlasov et al., 1974). The weak point of the results of correlations is that attempts are made to find a correspondence between frequency spectra of strictly periodic events (planetary configurations) and periodicities with disturbances (manifestations of solar activity). Even if the needed periods are revealed, either phase correlations often are absent (Dolginov et al., 1972) or it is impossible to explain irregular variations of momentary values of solar periods. Moreover, certain dissatisfaction is also caused by that fact that different authors succeed in describing the run of solar activity in the same time interval by means of the action of different planetary combinations. The negative result of the Schuster test application to verify the conditionality of flare activity by the action of Venus and Jupiter can be set off against the link of separate important phenomena of solar activity with planetary configurations (Dingle et al., 1973). Concrete physical mechanisms, apart from Dauvillier $(1970,1973)$, have not been proposed by anybody. Makarenko (1973) proved the possibility of the existence of a cybernetic model of a 'black box with input' in which the frequency trapping is realized, for the Sun, in an extremely abstract way but without indicating the physical mechanisms. This result is of purely academic interest only. 
So, the hypothesis of the planetary effect on solar activity is not more than a working hypothesis, but a detailed and objective analysis of the reality and nature of the correlations, is needed.

One should dwell separately on the ideas developed in a number of papers by Vasil'eva and her colleagues. According to these studies, a predominant direction exists in space. It corresponds to projections at the ecliptic of the direction to the galactic center, and of the direction to the standard apex of the Sun's motion, and to the direction of the large-scale galactic magnetic field. The authors postulate the electromagnetic character of the interaction between the planets and the Sun with due regard for the magnetic field of the Galaxy. Hence, the problem of planetary action on solar activity is transformed into the problem of the galactic action on solar activity, through the means of the planets. One of the possible mechanisms is the connection with a variation of the EMF produced under the influence of the external galactic field in a closed conductive circuit formed by the Sun, a planet and the interplanetary medium plasma with the frozen-in regular magnetic field of solar origin. However, all correlations of EMF changes with the variation of the solar activity level have again a conventional character due to a great number of assumptions, and in this sense they differ little from similar papers by other authors.

\subsection{SOME GENERAL CONSIDERATIONS}

It is easy to calculate that the energy of solar rotation is $10^{38} \mathrm{erg}$ (all estimations are made for 24-hour interval). The energy of convective motions in the subphotosphere layer of order $20000 \mathrm{~km}$ thick, where the major part of magnetic energy associated with sunspots seems likely to be concentrated, is equal to $5 \times 10^{36} \mathrm{erg}$. Of the same order is the energy of differential rotation. The estimation of the magnetic energy of sunspots has the form $8 \times 10^{31} f_{0} T_{0}$ erg. Thus, magnetic energy from minimum to maximum of the 11-year cycle varies on the average, from $10^{31}$ to $14 \times 10^{31} \mathrm{erg}$. If the magnetic energy of sunspots is assumed to be a result of mechanical energy transformation, for mechanisms related to either convective motions or differential rotation, the efficiency proves very small, of order $10^{-4}-10^{-3}$. With respect to tidal forces it does not exceed $10^{-3}$. We can consider the sources of mechanical energy to be inexhaustible. The losses in magnetic energy and mechanical energy carried away in the main by the solar wind, are correspondingly estimated by the values $10^{30}$ and $10^{32} \mathrm{erg}$. In the simplest form, the equations of energy balance have the form:

$$
\begin{aligned}
& \frac{\mathrm{d}}{\mathrm{d} t} E_{k}=Q-F\left(E_{k}, E_{m}\right)-\lambda E_{k}, \\
& \frac{\mathrm{d}}{\mathrm{d} t} E_{m}=F\left(E_{k}, E_{m}\right)-\mu E_{m},
\end{aligned}
$$

where $Q$ is the source of mechanical energy, $F\left(E_{k}, E_{m}\right)$ is the term describing the transformation of mechanical energy into magnetic energy, and $\lambda E_{k}$ and $\mu E_{m}$ are corresponding losses owing to the solar wind. The speed of magnetic energy changes is $8 \times 10^{31} f_{0} \mathrm{erg}$, varying during a cycle on the average from $10^{31}$ to $16 \times 10^{31} \mathrm{erg}$ per day. Unfortunately, estimations of the rate of change of mechanical energy are highly 
uncertain. Because a limit exists for sunspot dimensions and for the number of very large sunspots, it is evident that $F\left(E_{k}, E_{m}\right)$ is limited and has a single maximum.

All terms of our equations are positive, and a qualitative analysis shows that the system described by the equations must have a stable node. Really, the simplest auto-oscillation system with relaxation oscillations is described by Van der Pole's equations:

$$
\dot{x}=y, \dot{y}=-x+\varepsilon f(x, y), \quad f(x, y)=y\left(1-x^{2}\right)
$$

different from our equations. In order to produce oscillations $E_{m}$ it is necessary for either the system to be non-autonomous, i.e. subjected to an external influence, or phenomena of parametric resonance must be realized in it. Essentially, dynamo mechanisms precisely are one of the cases of parametric resonance manifestations. There is no doubt that the first equation 'does not' depend on $E_{m}$. Conversely, it is the governing equation. Thus even very small variations of the $E_{k}$ speed change may cause considerable variations of $E_{m}$. It follows from the above that the problem of solar activity mechanism autonomy is not an artificial one and needs particular attention and critical analysis.

As regards the physical nature of the secular cycle, only the hypothesis by Kopecký (1964) has been suggested about the possible existence of variations of the subphotospheric layer characteristics within the secular cycle where magnetic field tubes are formed. Dmitrieva et al. (1971) have shown that the sunspot distribution in respect to area varies regularly from the 15 to the 18 th cycle, and the variations of some importance indices is caused by this. Kuklin (1973) has shown that the variations of a number of importance indices from cycle to cycle are linearly dependent. On can introduce a certain parameter, $q$, determined with an accuracy up to linear transformation on which many importance indices depend linearly. This parameter, naturally, varies with the secular cycle, although probably, it has a weak 11-year variation. The simplest interpretation suggests the existence of two relatively stable sunspot populations, the number ratio of which varies with the secular cycle. Population II contains predominantly small sunspots and sunspots of increased stability, the magnetic field in them being stronger. The fraction of Population II is larger during the minimum epoch of the secular cycle. Population I represents the common sunspot mass. One may interpret secular variations as cyclic variations in an original ecologic system where competition exists between two populations. The introduction of a 'universal' $q$ importance parameter stimulates the need for a more detailed study of its significance, sense and properties.

\section{Facts}

\subsection{Supersecular CYCles}

It is rather difficult to say how far the solar activity cycles hierarchy extends to long periods. A sufficiently detailed list of basic cycles of the principal phenomena in the atmosphere, hydrosphere and lithosphere in connection with solar activity is given by Eigenson (1963b). Analysis of the complex of geophysical, geological and geographical phenomena allows us to assert that a cycle of 1700-1900-year length 


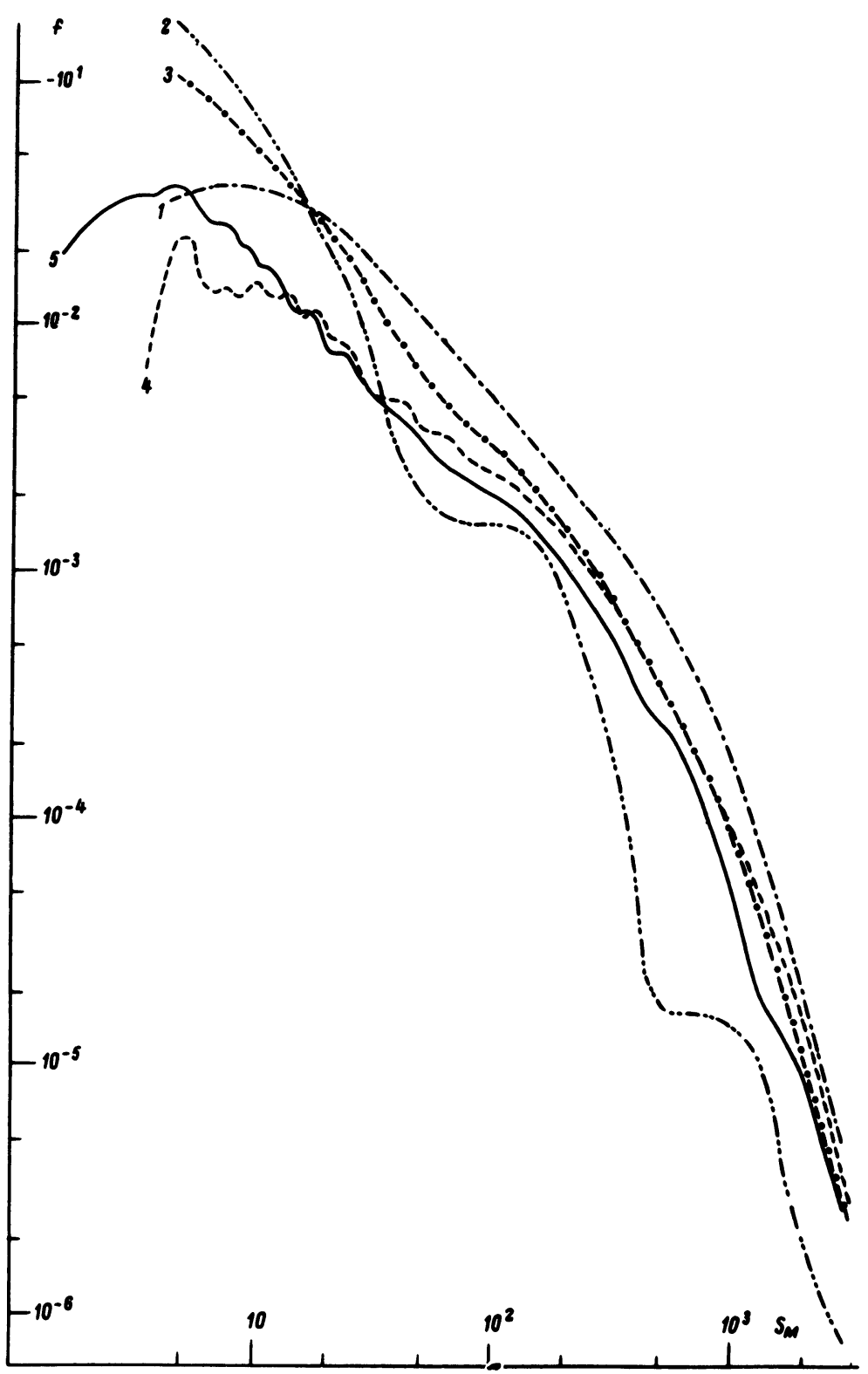

Fig. 4. The sunspot distribution in respect to the maximal area $S_{m}$ for the Population I (1) and II (2). 


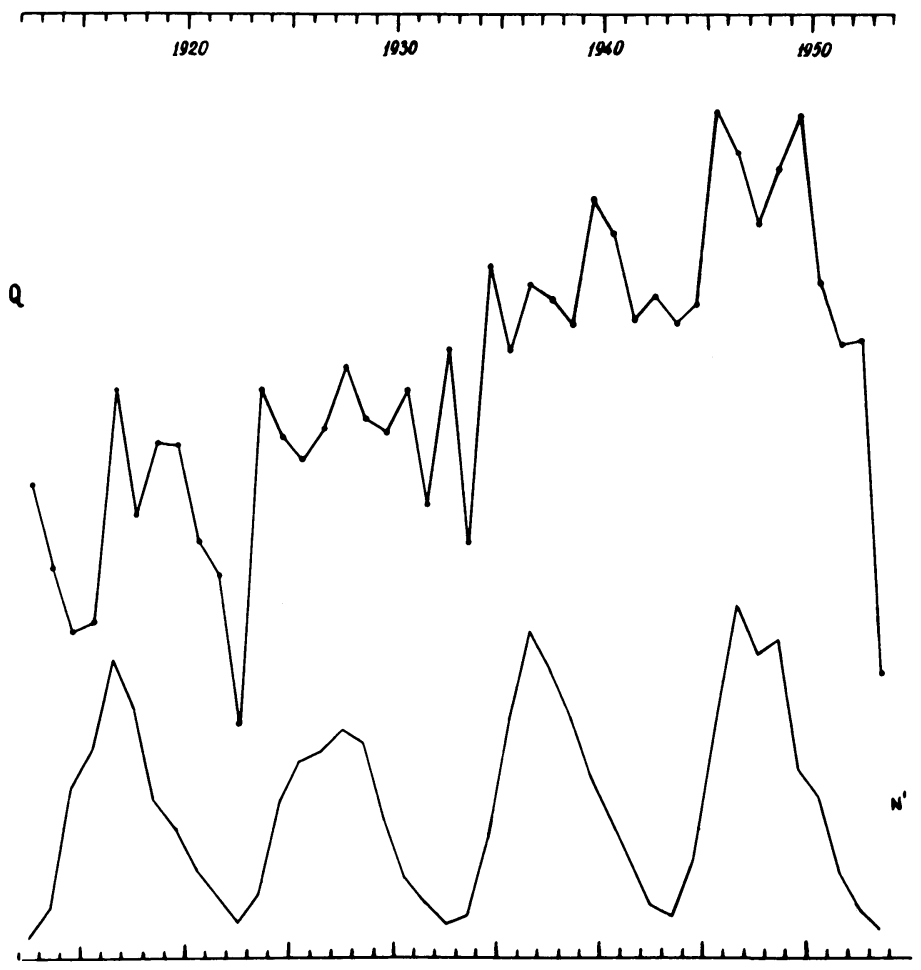

Fig. 5. The time-series of $q$ parameter values and of their relative weights $n^{\prime}$.

exists, Shnitnikov (1951) considering changes of continent moistenings which, according to his opinion, are caused by solar-terrestrial relationships, concluded that such a cycle ought to be present in the solar activity level variations. Aside from questions about the existence of a significant influence of solar activity on terrestrial processes, we also can consider such a cycle to be quite real. However, in this case all information is reduced to a statement of the fact that the cycle of such duration exists.

Rubashev (1949) examined the catalogue of comets visible with the naked eye and concluded that a solar activity cycle of nearly 900 -year duration exists. The study of 11-year cycle lengths on the basis of Schove catalogue allowed Henkel (1972) and earlier Golubtsov (1965) to discover this cycle. Also Gleissberg mentions the existence of this cycle as a result of study of the asymmetry index of 11-year cycles.

A solar activity cycle of nearly 600-year duration reveals itself by a study of comet number (Rubashev, 1949), by a study of 80-year cycle amplitudes using the width of tree rings (Maksimov, 1952), and by a study of the variability of the relationship between 11-year cycle height $W_{m}$ and its ascending branch length $T_{A}$ (Waldmeier, 1966; Vitinsky, 1969c). Golubtsov (1965) has obtained an estimation of $650 \mathrm{yr}$ for a cycle consisting of two semicycles, $325 \mathrm{yr}$ each. This cycle has proved itself by forecasts of activity (Vitinsky, 1973c). Besides, there are indications that this cycle manifests itself in hydrological, geological and climatic processes. 
Link (1963) has revealed a cycle of 400-year duration using data on climate changes, the numbers of discovered comets, and aurorae, and the secular variations of geomagnetism. It was found that the 80 -year cycle length is longer at its ascending branch.

The data on aurorae number and climatic changes are evidently in favour of the existence of a 300-year cycle but it is difficult to state direct arguments following from solar activity changes. However, Kolomiets has apparently found an 11-year cycle length variation within a period of 32 cycles (1969). It ought to be shown that if both the values of certain index and its change in speed (dynamical aspect) are important, then any cycle of duration $T$ must give rise to cycles of duration $T / 2$.

Predtechensky (1948) on the basis of a study of alternation regularities in the form of the cycle proposed a hypothesis on the existence of a 189-year period called 'indiktion' and containing 1711 -year cycles, but there are no serious reasons to take this hypothesis into consideration (Rubashev, 1969).

In a number of papers by Bonov (1957 and so on) a cycle of 176-year length (16 11-year cycles) was studied. It manifests itself in variations of a number of 11-year cycle pair characteristics: the length ratio even-odd, the sum of the ascending branch length etc. Bonov (1973) comes to the conclusion that such a cycle must begin from an even-odd pair and at the borders of it the solar activity level falls unevenly. Vertlib and Kuklin (1971c) studying the neighboring cycle pair links found the 176-year period also.

Vasilyev (1970) has revealed a 178-year period from the spectral analysis of Wolf numbers. According to Chirkov (1971) the total importance of the 22-year cycle $W_{M}$ varies within $8 \times 22 \mathrm{yr}$. One ought to note that doubts in these results were expressed since the initial data series is a little longer than the cycle length considered, although this cycle may be placed twice in the interval of the telescopic observations of the Sun.

An assumption on the existence of a cycle of 169-year length was suggested by Anderson (1954) and it must consist of two parts of 88- and 81-year duration. This cycle is traced in annual and monthly Wolf numbers. Djurkovic (1956) has discovered a cycle of 168-year length using data on the 11-year cycle extrema from 1610 . It is necessary to note that the spectral analysis also revealed a period of $168 \mathrm{yr}$ (Vasilyev and Vitinsky, 1969). The period of $169.0 \pm 0.5 \mathrm{yr}$ was found with the help of an autocorrelation function by Gabrovsky et al. $(1967,1968)$.

Apparently just now it is difficult to decide if the cycle of 169- or 176-year length is simply a double secular ( $80-90 \mathrm{yr})$ cycle or not but this is not important for the forecasting significance of these cycles. Particularly in the opinion of Eigenson and Mandrykina (1962b) when comparing the increasing branches, the current secular cycle is similar not to the preceding one but to the one before the preceding branch, which argues in favor of double secular cycle reality. However some authors suppose that these cycles are not independent. So Cohen and Lintz (1974) consider that the 179-year period arises as a result of beating at close frequencies $\sim 0.09 \mathrm{yr}^{-1}$ and $0.1 \mathrm{yr}^{-1}$ present in the spectrum. Analogously Cole (1973) has obtained the result that superposition of oscillations with derived periods of 10.45 and $11.8 \mathrm{yr}$ has a phase variation with a period $\sim 190 \mathrm{yr}$. 
Thus, it is permissible to speak about the presence of supersecular cycles of 600and 170-180-yr length which manifest themselves in a number of solar activity characteristics, and about the possible existence of other supersecular cycles.

\subsection{THE SECULAR CYClES}

The secular cycle, or 80-90-year cycle, sometimes gives rise still to doubts by individual authors concerning its reality, but we don't consider them. Even the first investigators of solar cyclic variations suspected its existence. Contributions to its study were made by Wolf, Gleissberg, Eigenson, Waldmeier and others. In the simplest manner it reveals itself when smoothing procedures are employed or envelope curves are constructed.

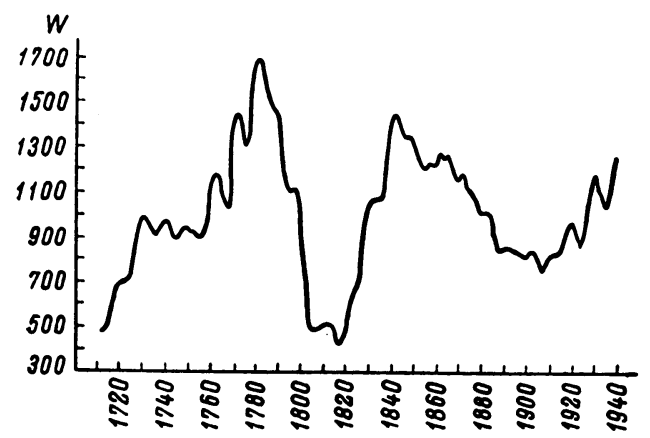

Fig. 6. The secular cycle of Wolf numbers in case of 23-year sliding summation.

The properties of the secular cycle determined from Wolf numbers differ in great extent from the 11-year cycle properties. The length of the secular cycle fluctuates within broad limits from 20 to $130 \mathrm{yr}$ with an average duration of about $80 \mathrm{yr}$. In different centuries the secular cycle is unequally sharp; Sometimes it does not reveal itself at all. Spectral analysis (Vasilyev and Kandaurova, 1968, 1970a, b) provides a 91.3-year estimate for the period. According to Rubashev (1964) the correlation between secular cycle in principle represents a pseudo-harmonic variation, the first year after the descending branch length also increases with the cycle importance, but this relationship is more weak. Correlations will be higher if the secular cycle importance is estimated by the difference in activity levels (Gleissberg, 1966). The importance ratio in maximum and minimum is of order 3. Vitinsky (1971a) has shown that the secular cycle in principle represents a pseudo-harmonic variation, the first year after the 11-year cycle maximum being important for the secular cycle. The extreme epochs of secular cycles are closer to those of the 11-year cycle maxima (Vitinsky, 1968a). Chistyakov (1963) on the basis of a mirror symmetry of secular cycle branches found by him, believes that a secular cycle begins and ends with a maximum.

The secular cycle reveals itself in variation of different characteristics of the 22-year cycles (Bonov, 1964), 11-year cycle asymmetry index (Eigenson and Mandrykina, 1962c), 11-year cycle length (Henkel, 1972; Kolomiets, 1969), jerk index (Vitinsky, 
1968c). According to Chistyakov's studies (1973), secular cycles reveal themselves more on those 11-year cycle phases when the mean sunspot zone latitude is close to $16^{\circ}$. Afanasyev (1961) concluded that according to the 11-year cycle mean importance index $\sum W / T$ on the secular cycle ascending branch, pairs are formed in combination (low, odd, high, even), and on the descending branch in the reverse way. The length of the 22-year cycle determined with regard for superimposition varies with the secular cycle too (Chistyakov, 1961). Studying the break-points in 11-year cycle curves Chistyakov concluded that only points $k_{2}$ and $t$ were associated with the secular cycle. As far as the same determined phases of the most important cycle manifestation, this once more supports the secular cycle interpretation as an importance cycle.

Actually, one can easily make sure of the latter as we shall show now in the consideration of other characteristics of solar activity. Because regular observations of sunspot areas cover only $100 \mathrm{yr}$, the reliability of determining the secular cycle length from phenomena importance indices is small, but it is easy to show their variations to have a secular cycle character. The difficulty of determining the secular cycle length from available importance indices series is illustrated by the results obtained by Vasilyev and Vitinsky (1969), where estimates are obtained within 55-95 yr. According to Kopecký (1967) a great number of indices have such variations: mean sunspot group lifetime, mean sunspot group area, recurrence index, mean maximum sunspot group area, and maximum being more than $1000 \times 10^{-6}$. A secular

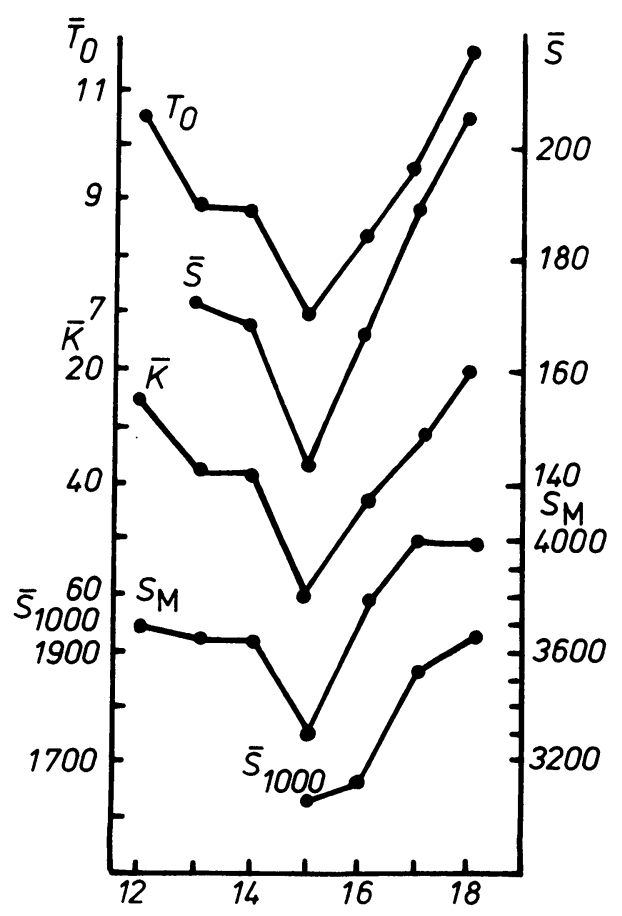

Fig. 7. The secular cycle of importance indices according to Kopecký. 
variation is present in the one-day sunspot group number - total sunspot group number ratio (Ringnes, 1962), mean magnetic field strength (Ringnes, 1965), regression equation coefficients $H-S$ (Ringnes, 1965), sunspot penumbra area - sunspot umbra area ratio (Ringnes, 1965b), mean sunspot group latitude (Ringnes, 1968; Kuklin, 1971b), high-latitude of sunspot formation (Gleissberg, 1958), high-latitude sunspot group number (Kopecký, 1958; Waldmeier, 1966), and fluctuation duration in the 11-year cycle descending branch (Vitinsky and Ikhsanov, 1970).

The attempts to present the secular cycle as a result of oscillation beating with close periods using only the analysis of Wolf number series seem to be somewhat naive (Pavelyev and Pavelyeva, 1965; Cheng and In, 1965; Cole, 1973; Zhukov and Muzalevsky, 1969; Muzalevsky and Zhukov, 1968a, b). These results should be attributed to the category of erroneous ones. It should also be noted that the parameter $q$, figuring in the concept of two populations of sunspot groups (Kuklin, 1973) since it is inferred from phenomena importance indices, also must vary with the secular cycle. In the framework of this concept the secular variation of precisely such characteristics does cause similar changes of all the above indices.

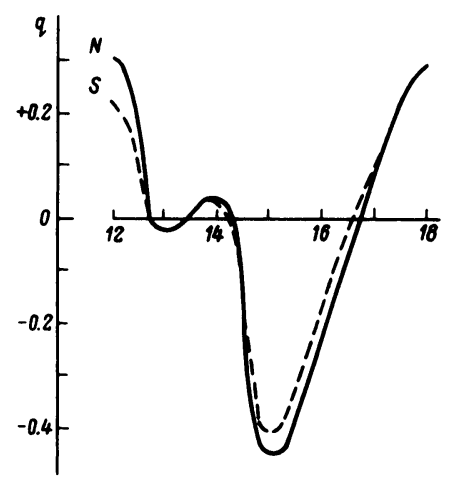

Fig. 8. The secular cycle of $q$ parameter.

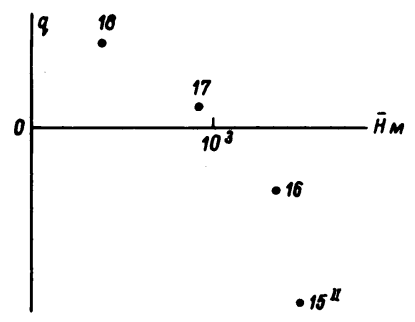

Fig. 9. The dependence of the mean magnetic field strength on the $q$ parameter.

Asymmetry of the $N$ and $S$ hemispheres of the Sun manifests itself in the difference in shape between 11-year cycle curves and extremum epochs in the surplus of summarized area and sunspot group number in either hemisphere. 
For a given characteristic $C$, separately determined in both hemispheres, one can introduce two nearly equivalent asymmetry indices

$$
A_{c}=\frac{C_{\mathrm{N}}}{C_{\mathrm{S}}}, \quad a_{c}=\frac{C_{\mathrm{N}}-C_{\mathrm{S}}}{C_{\mathrm{N}}+C_{\mathrm{S}}}
$$

Waldmeier (1957) investigated in detail the asymmetry between the hemispheres total sunspot group area. According to his results this asymmetry has a secular cycle (correct oscillation) with $N$ hemisphere predominant in minimum and the $S$ one in maximum. Ringnes (1968) considered the asymmetry of hemispheres using the mean sunspot latitude. The schemes of asymmetry manifestation according to Waldmeier and Ringnes are presented in Figures 10 and 11. It is curious to note that variations in
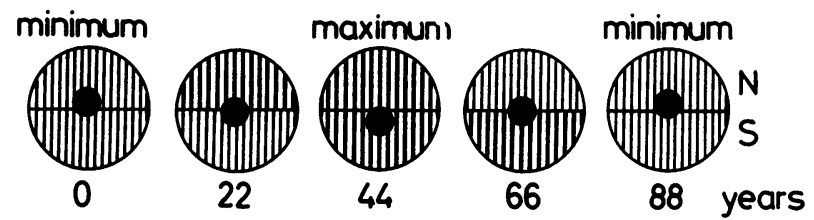

Fig. 10. The scheme of the N-S asymmetry variation during the secular cycle according to Waldmeier.

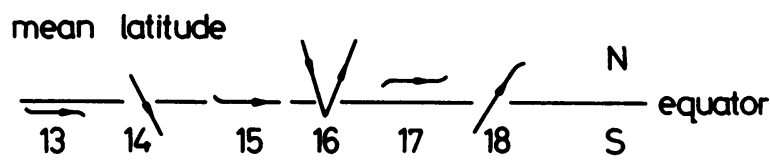

Fig. 11. The scheme of the mean sunspot latitude variation during the secular cycle according to Ringnes.

mean latitude are opposite in high latitude sunspot groups in comparison with low latitude ones. However, one should pay attention to the fact that in cycles 19 and 20 a predominant development of activity was observed in the $\mathrm{N}$ hemisphere in disagreement with Waldmeier's scheme. The cause for such a marked and long-stable one-sign asymmetry is worthy of a special study.

Berdichevskaya (1967) considered the area-weighted latitude of the first sunspot groups at the beginning of 11-year cycles. These characteristics show a 55-year cycle periodicity, the $\mathrm{N}$ hemisphere being predominant in the minimum of the current secular cycle.

It should be noted that in a number of values the secular cycle appears as a cycle of about 55-year duration. The same period reveals itself in a spectral analysis (Vasilyev and Kandaurova, 1968). In connection with the secular cycle it is necessary to mention the study by Bezrukova (1968), where it is found that cyclic curves of sunspot group area sums averaged during their appearance in one and the same hemisphere are more similar every 4 cycles, i.e. every 44 yr. Besides, Trellis (1973) found that the duration of activity region displacements from mean positions varies with an approximately 55-year cycle. 
Therefore, the secular cycle exists in reality, it is associated predominantly with important manifestations of solar activity and asymmetry effects and has properties different from those of the 11-year cycle.

\subsection{THE 22-YEAR CYCLE}

The 22-year cycle became recognized only after Hale's discovery of the law of sunspot magnetic field polarity changes, although earlier investigations of Wolf and Turner show indications of the existence of such a cycle. This cycle reveals itself more sharply in magnetic characteristics and therefore is more physical. However, it is harder to find using other indices, therefore it is more just to be considered in principle as a cycle of qualitative characteristics of solar activity.

Above all, the 22-year cycle manifests itself in forming 11-year cycle pairs according to the Gnevyshev-Ol' rule (1948). Where the statistical relationship between 11-year characteristics is more close in the even-odd combination. This refers to Wolf number sums (Gnevyshev and Ol', 1948), to the relationship between the length of the 22-year cycle and the interval between 11-year cycle maxima of a pair (Bonov, 1958), and 11-year cycle relative height in a pair (Rubashev, 1964). Essential deviations from obtained correlations fall on secular cycle extremum epochs (Chistyakov, 1959b).

Turner as long ago as 1925 emphasized that in odd 11-year cycles, the sunspot areas are larger and their latitude is $1^{\circ}$ higher in comparison with even cycles. Usually an even cycle is lower and this regularity is more evident in the behavior of the new sunspot group appearance (Kopecký, 1958, 1967). The 22-year cycle length decreases with the growth of its importance (Bonov, 1958; Chistyakov, 1965; Chirkov, 1971). An analysis of cyclic curves (Vertlib and Kuklin, 1971c) supported the Gnevyshev-Ol' rule and showed a difference, in this sense, between even and odd 11-year cycles. Investigating the break points Chistyakov (1965) found that there is a rather close relationship in the pairs for values corresponding to the points $k_{2}$ and $t$. However, in the odd-even combination, characteristics of nearly all break points exhibit high correlation to the 22-year cycle length. These facts as well as the results by Afanasyev (1961) seem to speak well for the hypothesis that it is not important what the cycle order is in a pair, but interchange of properties is important in neighboring 11-year cycles (Chistyakov, 1973).

The study of cyclic curves of different indices in separate latitude zones (Vitinsky, 1965a) shows a weak 22-year cyclic periodicity. Vasilyev and Vitinsky (1959) did not succeed in clearly revealing the 22-year cyclic periodicity for phenomena frequency indices which to a certain degree contradicts the results by Kopecký (1958) and Figure 2.

The sign of the Sun's polar cap magnetic field changes with the 22-year length cycle and the same for the coupling character between increased brightness regions of the green corona and the sector structure of the IMF (Antonucci, 1974). Vasilyev and Rubashev (1972) have established that variations of solar radius occur with the 22 -year cycle. The radius, in even cycles, increases with a velocity varying in parallel to the Wolf number run, and decreases in odd cycles. The coupling between neighboring 22-year cycles is reduced to that with increasing preceding odd cycle, the 
maximum of the next even cycles comes later, the length of the next 22-year cycle is longer and its importance is less (Chistyakov, 1959a). Many authors attach great importance to the 22-year cycle in view of the fact that the latter in its nature, is a magnetic cycle. For this reason, attempts are continued to operate with Wolf number sign-changing series (Anderson, 1938; papers by Muzalevsky and Zhukov). Vasilyev and Vitinsky (1969) showed that these formal operations in principle do not yield any new or better results, although it is tempting to explain rich spectra by the presence of a small number of basic periods. Nevertheless, periods close to 22 years, are found in almost all papers on frequency spectra of solar activity.

On the other hand, there is no sense in disregarding the reality of the 22-year cycle. For this reason, any physical and mathematical models of solar activity must lead to the basic period of order $22 \mathrm{yr}$. At the same time all variants of dynamo-mechanisms satisfy this. Apparently, this was sure to be the direction of models considered in a series of papers by Gudzenko and colleagues. From this viewpoint, the eruptive hypothesis of independent 11-year cycles raises objections.

The change of sunspot magnetic field polarities suggests an idea on the relationship between hemispheres in neighboring cycles. In particular, such a coupling follows from the Alfvén mechanism of solar activity (1945a, b). Recently (Fredga, 1965), the presence of such a coupling was supported when considering the similarity of sunspot distributions in latitude intervals at the beginning of each cycle. It is curious that on the 'latitude-time' diagram, single fluctuations with 1-year resolution form chains which, being extended into another hemisphere and another cycle, indicate either the beginning or maximum of similar chains.

An original concept of the magnetic cycle was suggested by Ol' (1972). He places emphasis on the coupling between geomagnetic recurrent disturbances on the descending branch of a previous cycle, and the characteristics of the oncoming cycle maximum. Taking into account the correlation for even-odd cycles, Ol' comes to the following scheme (Figure 12). The cycle of solar magnetic activity begins with the development of unipolar magnetic regions at the end of the old cycle which induce the development of unipolar and bipolar regions in the even cycle, and it ends with

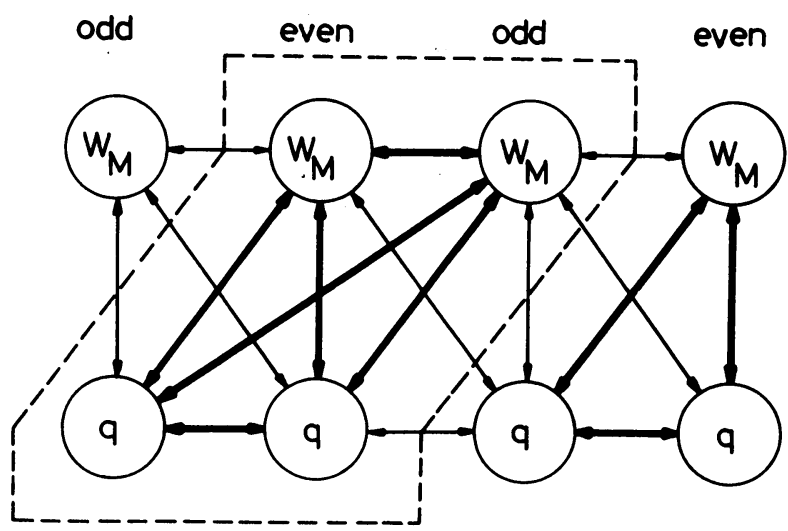

Fig. 12. The cycle of magnetic activity according to Ol'. 
the development of bipolar regions in the next even cycle. The length of such a cycle, with due regard for superposition, ought to be about 26 yr. Finally, if a cause-effect interaction between the laws of Hale and Gnevyshev-Ol' (physical and statistical aspects of 22-year cyclic periodicity) is postulated, then one should expect that violations of the second law caused by the secular and supersecular cycles, must correspond to violations of the first one. Otherwise, the expected violation of the Gnevyshev-Ol' law in the pair of cycles 20-21 must be accompanied by the absence of a magnetic field polarity change.

Hence, the 22-year cycle is mainly associated with the variation of magnetic sign characteristics and secondly it appears only in frequency or importance indices. Besides, $\mathrm{N}-\mathrm{S}$ asymmetry does not seem to be related to the 22-year cycle (Vasilyev and Vitinsky, 1969).

\subsection{THE 11-YeAR CYCLE}

We shall not consider now the list of the main peculiarities of the well-known 11-year cycle of solar activity but in the first instance we shall focus our attention on its less known properties and manifestations.

It is known that the granule number on the Sun varies in parallel with the run of Wolf numbers (Macris, 1960). The intensity of Fraunhofer lines in the spectrum of the undisturbed photosphere also depends on the cycle phase: the central intensity is a minimum in years of maximum (Zhukova and Mitrofanova, 1973).

Somewhat contradictory information has been obtained using sunspot structure variations with the cycle phase. Deszö and Gerlie (1964a, b, 1965) found a cyclic variation of the penumbra-umbra area ratio. According to their data this index consecutively decreases from minimum to maximum of the cycle showing on the descending branch values greater than those on the ascending one. Highly detailed investigations of this index were performed by Antalova (1971) who came to the conclusion that it is an increasing function of Wolf number, i.e. it repeats, the shape of the cyclic curve. In the epoch of the secondary maximum the values of the index usually are less than in the epoch of basic maximum. The index is, essentially, a function of only cycle phase and sunspot area.

At the counter-phase polar plages (Sheeley, 1964), polar prominences (Godoli and Mazzuconi, 1967) as well as intensities of weak magnetic fields in polar caps (Howard, 1965) vary with the run of Wolf number during the cycle. It is considered that the background fields of a previous cycle with related plages and prominences reach polar regions with considerable delay of more than half a cycle and by that time magnetic fields of a new cycle have time to appear at intermediate latitudes. These data are an indication of the existence, at high latitudes $\varphi>40-45^{\circ}$, of another ring of meridional circulation directed towards the pole in the photosphere. It is of interest that in cycle 20 a secondary zone of polar prominences within 1968-1971 was observed (Waldmeier, 1973). Simultaneously, a discontinuous displacement of the sunspot production zone towards the poles was noted. Such incidents in operation of the cycle machine are interesting and need detailed study.

The coronal emission in the red line of $6374 \AA$ does not undergo considerable cycle changes but the emission intensity of the green line of $5303 \AA$ exhibits cyclic 


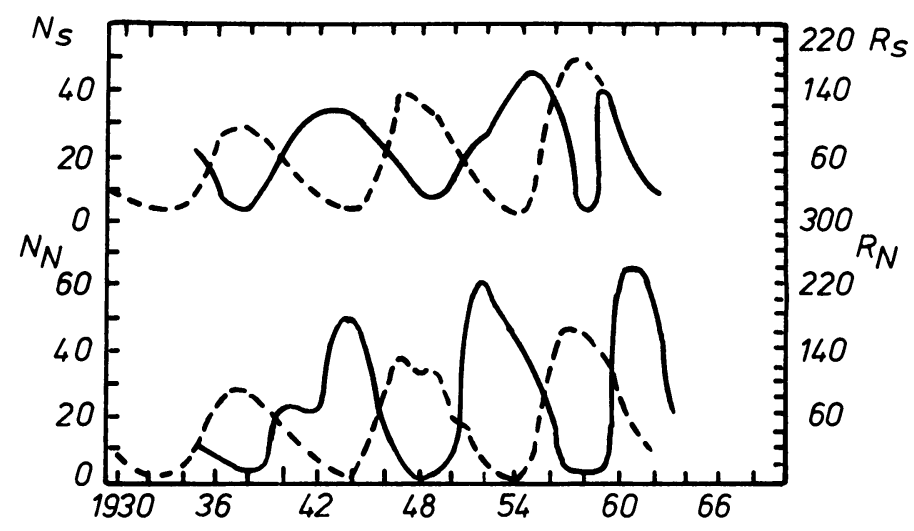

Fig. 13. The cyclic curve of the polar faculae number according to Sheelly.

variation. Because the regions of increased coronal brightness coincide with solar activity phenomena, this allowed Gnevyshev to detect secondary maxima of the 11 -year cycles $(1963,1966,1967 a)$. The $\mathrm{N}-\mathrm{S}$ asymmetry index of green corona brightness also has two maxima and exhibits negative correlation with the sunspot number (Pathak, 1972). As has often been mentioned, the secondary maxima are caused by the increasing share of importance phenomena of solar activity. Therefore, the secondary maxima are found on cyclic curves of the number of type-IV radio bursts and the S-component level (Křivský and Krüger, 1966), of important calcium flocculi (Basu and Das Gupta, 1966), and of proton flares (Gnevyshev and Křivský, 1966). More correctly, the basic maxima of these cyclic curves lag 1-2 yr with respect to the cyclic maximum and coincide in time with the secondary Wolf number maxima.

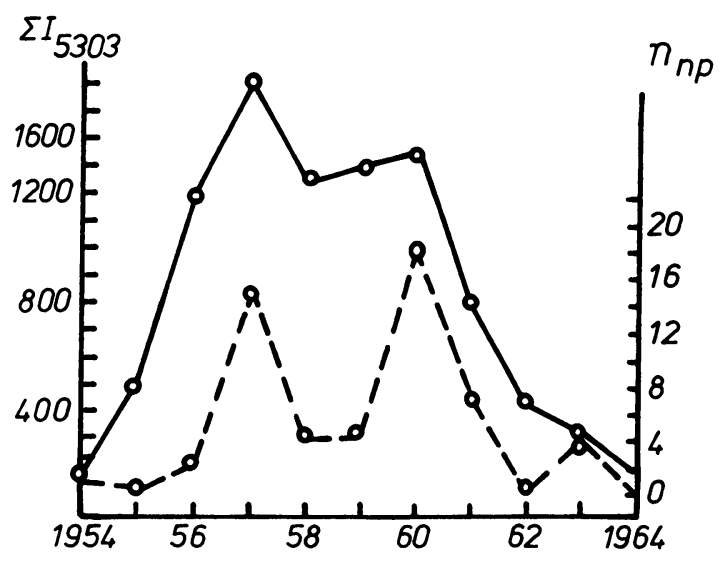

Fig. 14. The cycle curve of the green corona emission (solid line) and of the proton flare number (dashed line) according to Gnevyshev. 
If we operate with sunspot, plage, flocculi, flare, and prominence numbers irrespective of their importance, the corresponding cyclic curves differ little from Wolf number curves (Rubashev, 1964). Zabza $(1962,1964)$ has found that the following index combinations have conformal variations during a cycle: sunspot area - flare index - radio emission flux $\lambda=10.7 \mathrm{~cm}$, red corona brightness - radio emission flux $\lambda=10.7 \mathrm{~cm}$, red corona brightness - prominence index.

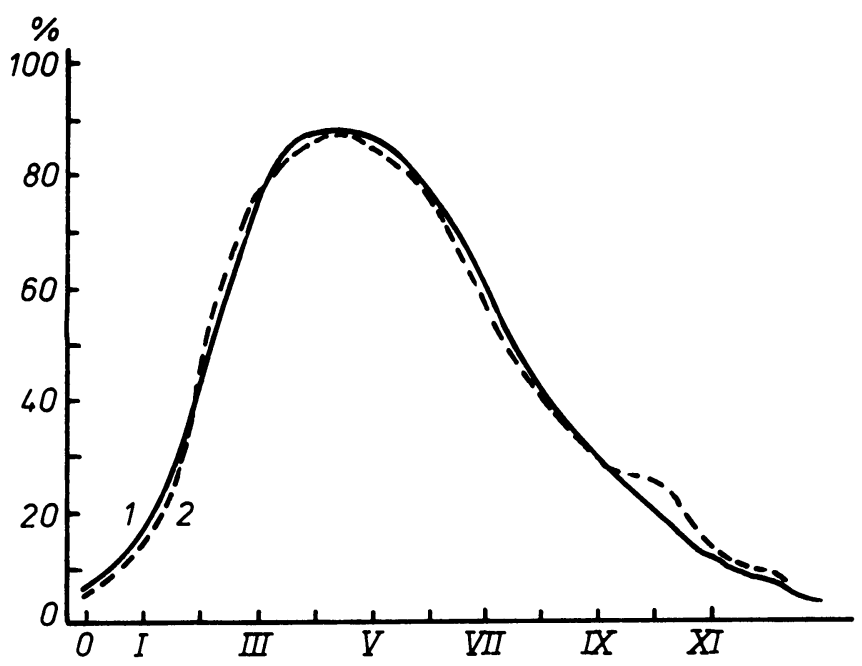

Fig. 15. The cycle of Wolf numbers.

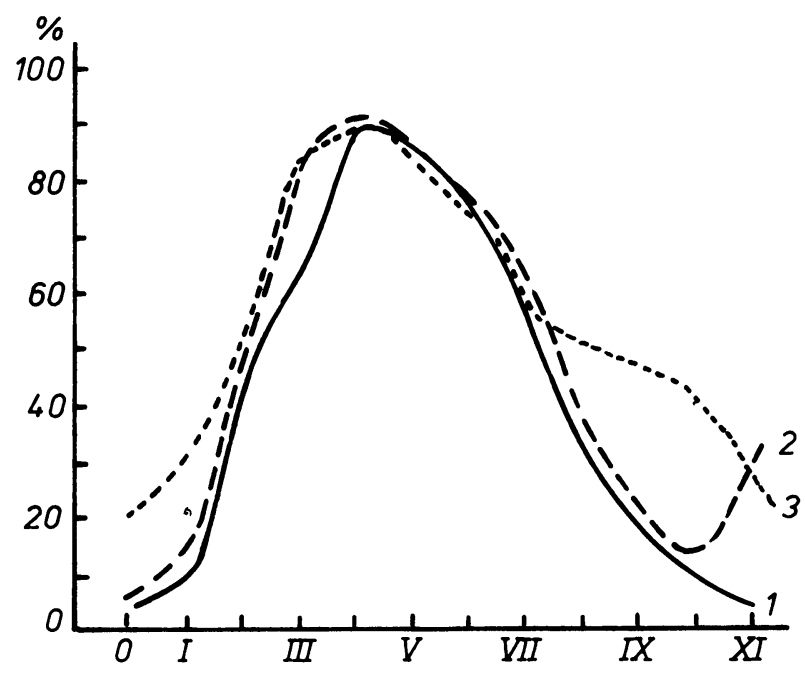

Fig. 16. The cyclic curves of the sunspot area (1), the faculae areas (2), the prominences areas (3). 
According to Fokker (19.63), small microwave bursts are encountered equally often, independently of the cycle phase, but large bursts prefer to concentrate on the descending branch where they are encountered more often than in the cycle maximum.

As mentioned, the number of important proton flares has a maximum after 1-2 yr of the maximum but for proton flares of small importance (class 1 and 2) a simple increase of their part to minimum is typical (Levitsky, 1967b) as well as for flares accompanied by type IV bursts.

The fluxes of X-ray emission during the epoch of cycle maximum, in comparison with the epoch of minimum, increase 20 times in the 44-60 $\AA$ wavelength range and 200 times in the 8-20 $\AA$ wavelength range (Kreplin, 1970). It should be taken into account that estimates of magnetic energy, calculated for sunspots only vary by about 16 times.

Wilcox and Colburn (1069) emphasize that the IMF sector structure on the increasing branch is characterized by considerable instability.

According to Hakura (1974), geoefficiency of the Sun during the 11-year cycle three times reaches maximum values. The first maximum approximately coincides with the main cycle maximum or somewhat precedes it and is associated with the maximum of relatively small phenomena number on the Sun. The second maximum enters 1-2 yr later than the main one and corresponds to the maximum of important phenomena number on the Sun. Finally, the third maximum comes 4-5 yr after the main one when on the Sun there is a maximum of unipolar long-lived regions responsible for a great number of recurrent disturbances.

So, additional data emphasize the peculiarity of the 11-year cycle that indices of phenomena frequency with no regard to their importance have the usual cyclic curves, and importance indices of phenomena have less marked 11-year variation with displaced maxima.

It is typical for the 11-year cycle that the ascending branch, i.e. its length, is of prime significance. This was mentioned more than once by Eigenson and Mandrykina (1962a). In a number of papers by Xanthakis he succeeded in constructing a family of formulae, permitting him to express many cycle parameters through this value and to give empirical formulae of its computation, using the ordinal number of the cycle. Unfortunately, they are very bulky and formal in their nature. The determining significance of the ascending branch length means that mathematical models of the 11-year cycle ideally seem to be one-parametric ones, and cyclic curves are likely to be close to relaxation oscillations. Hence, the basic ideas of the papers by Gudzenko and colleagues cannot be simply discarded, there is still some value in them.

Although Vitinsky (1972) has come to a conclusion that it is extremely difficult to reveal among the two tens of known cycles a pair of analogous cycles, similar to the extent that one can make predictions, nevertheless, the search for similarity is of some interest for a more general interpretation. Vertlib and Kuklin (1971a,b) employed for these purposes both a generalization of the method of principal components and non-orthogonal expansions. It turned out that two factors play their part in the difference between cyclic curves: the cycle height and the curve shape. 
One can reveal typical and individual cycles, using the latter factor. Typical cycles are divided into threes and such groups follow every 6 cycles.

Chistyakov (1965) has established a number of empirical correlations for 5 inflection points, found by him on the cyclic curve. These correlations, determining the shape of the cyclic curve, are helpful for predictions. It is important that the time interval between break points $S$ and $U$ is almost constant and is as large as $6 \mathrm{yr}$. With the help of this the cycle 'core' is detected, in which nearly all cycle importance is concentrated. The inflection points demarcate separate parts of the cyclic curve, within the limits of each part its own behaviour of fluctuations is established.

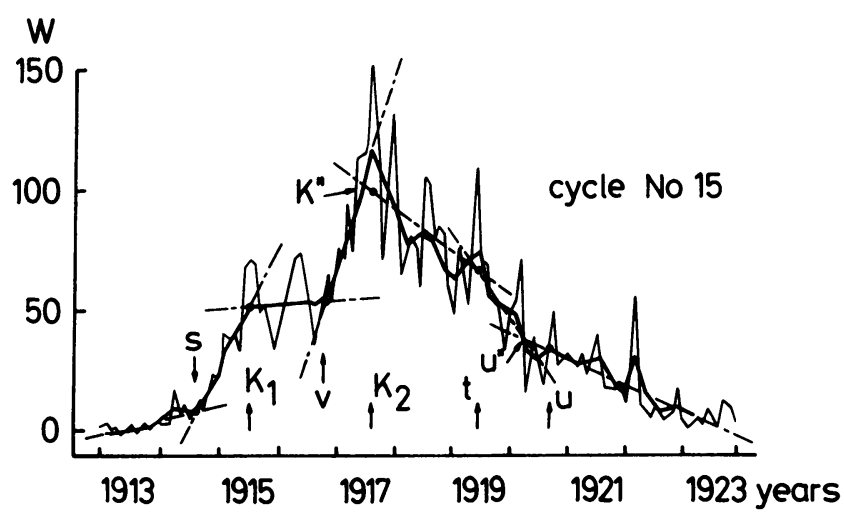

Fig. 17. The break points at the cyclic curve according to Chistyakov.

The result by Giovanelli (1964) is of interest not only for forecasting. It is shown that in the minimum epoch the curves of appearing sunspot group numbers are the same for all cycles when descending and ascending branches are examined separately. Szymansky (1974) has concluded that stabilization of cycle length takes place during several cycles as well as the correlation of intervals between cycles and their importance difference exists.

\subsection{THE SEASONAL VARIATION}

In recent years the problem of the reality of solar activity seasonal variation acquired new sharpness in connection with the fact that the presence of this variation is considered a nearly decisive argument in favour of gravitational planetary influence upon the Sun.

Suda (1962) and Romanchuk (1963) have concluded that the existence of seasonal solar activity variation is beyond doubt. Loewe (1973) has also found a secular variation of plage area with a maximum in July and a minimum in November. The results by Kozelov $(1972,1975)$ and those by Kozelov and Mingaleva (1975) are more impressive. The distribution of proton and important flares observed exhibits a 

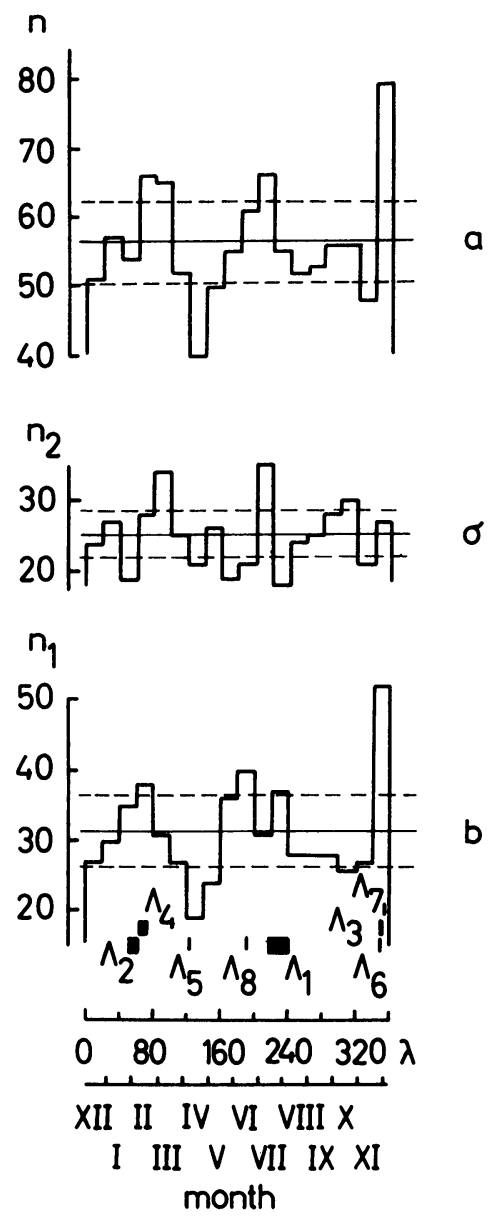

Fig. 18. The seasonal variation of the flare number and the resonance longitude location according to Kozelov.

non-random inhomogeneity with a probability more than 0.99 . The extrema of this polynodal distribution coincide with time intervals when the Earth has a longitude corresponding to resonance directions. It is curious that such a distribution is independent of activity level and is revealed from yearly repetition of geomagnetic disturbances which is effectively taken into account by a number of geophysicists in their planning of investigations. Finally, the seasonal variation of solar activity was revealed by Vasilyeva et al. (1974), but in contrast to papers by other authors, this variation turned out to be variable and dependent on cycle phase.

From papers of the opposite character one may quote the study by Vitinsky (1973a) in which the absence of any statistical significance of the obtained seasonal variation was proved after the analysis of the same material, used in the paper by Vasilyeva et al. Besides, Ambrož (1973b) has shown that the seasonal variation of Wolf numbers is unstable. 
The above information gives no reason to draw a final conclusion on the reality of seasonal variations. The fact of instability itself is no reason to deny flatly the existence of such variation. However, the results by Kozelov require this question to be approached extremely seriously and critically.

\subsection{Fluctuations}

Usually, fluctuations of solar activity are considered as relatively short-term (less than a year) deviations of index values from their smoothed values. Because the distinguishing of fluctuations implies the presence of a long uniform series of indices, the study of fluctuations up to now was based on data of Wolf numbers and total sunspot areas. Physically, fluctuations are caused by the appearance of activity centers, the life-time of which is comparable with the fluctuation length. The activity centers are the totality of related phenomena of solar activity, covering nearly all layers of the solar atmosphere and concentrated in a limited part of the solar surface.

Fluctuation indices may be constructed as differences or ratios of unsmoothed and smoothed values. Zhukov and Muzalevsky (1969) have shown that fluctuations of Wolf numbers may be considered as a multiplicative random process. In this case, fluctuation indices of the 'ratio' type will be free of 11-year cycle influence, concentrated in smoothed values. 'Difference' type indices are more subject to the influence of smoothed values but, nevertheless, they also bear useful information.

Vitinsky (1961a) has investigated in detail the properties of Wolf number fluctuations. He proved that fluctuations have an aperiodic character, i.e. they show no marked periodicity. As already mentioned, the 11-year cycle seldom manifests itself in fluctuations but the secular cycle is followed in a number of fluctuation characteristics, especially in their average duration. Here, the fluctuation length implies the interval between two neighboring maxima. The fluctuation character on the ascending and descending branches of the 11-year cycle is different, their amplitude and duration behaving less regularly on the descending branch. The relationship between the relative fluctuation amplitude and the ascending and descending branch lengths is positive and weak.

Parallel with the secular cycle in the fluctuation length on the descending branch of an 11-year cycle, a longer (double secular) cycle for the same value is revealed on the ascending branch of the 11-year cycle (Vitinsky and Ikhsanov, 1970). According to data of this investigation, $80 \%$ of the fluctuations are 2-4 months, and they are different on the ascending and descending branches. More short fluctuations with greater amplitude appear on the descending branch.

Fluctuations in amplitude, exceeding the mean square deviation, are called strong fluctuations. Vitinsky has made a catalogue of strong fluctuations (1960). There are more strong positive fluctuations on the longer ascending branch of the 11-year cycle. On the descending branch the density of these fluctuations depends on the cycle length and height. Strong positive fluctuations prefer the 1st year before the maximum and the 3rd year after the maximum. Strong negative fluctuations are more often encountered in the 1st, 2nd and 6th years after the cycle maximum (Vitinsky, 1963b). They, to a lesser extent, enter on the long ascending cycle branch. 
Chistyakov (1966) has found that $80 \%$ of the fluctuations in both hemispheres are synchronized. In the epoch of minima, fluctuations of the old and new cycle turn out to be synchronized to a considerable extent too (Chistyakov, 1968). A linear positive relationship exists between the fluctuation latitude of a new cycle and the fluctuation delay of the old cycle at low latitudes.

Dodson and Hedeman (1970) detected primary and secondary fluctuations of solar activity, related to activity centers. The mean time interval between primary fluctuations is about 15 rotations, and between the secondary ones - from 3 to 5 rotations. On the descending branch of the 11-year cycle 5-6 primary fluctuations are observed from which 2-3 are accompanied by the increased solar activity.

So, Wolf number fluctuations are independent random oscillations, manifesting themselves simultaneously in both hemispheres and possessing some regularities of lengths and amplitudes with respect to the 11-year cycle.

\subsection{LATITUDINAL DISTRIBUTION OF SOLAR ACTIVITY AND ITS DYNAMICS}

The distribution of solar activity phenomena in latitude is governed by Spoerer's law which says that during a cycle the zone of sunspot formation moves from a high latitude boundary to the equator. The character of the latitudinal drift, dependent upon the cycle phase, is of great importance for the construction of physical models of solar activity.

In the most general form Spoerer's law determines the variation of solar activity distribution in latitude with the cycle phase. An obvious idea of it is given by the 'latitude-time' diagram (Butterfly Diagram). Not a few papers are devoted to different aspects of the study of such diagrams.

The fine structure of the sunspot diagram was studied by Bell (1960) who concluded that the diagram represents a zonal pattern of sunspot formation ('caterpillars') without any latitude drift. Vitinsky (1961b) has proved the inconsistency of such conclusions. In recent years the fine structure of an index distribution in latitude with a period of order $1^{\circ}-2^{\circ}$ revealed itself (Granova et al., 1972; Kozhevnikov, 1973), but these results provoke the same objections. Kuleshova (1962) has come to the conclusion that 'butterflies' are composed of impulse-chains existing for 15-20 months and slipping down to the equator. Dmitrieva (1965), by special methods, has supported the reality of impulse-chains and has proved that those depend on the secular cycle phase and the longitude. Minasyants and Obashev (1969) have revealed the same impulse-chains for flares with life-time of about 12 months and drift velocity of order $1.8 \mathrm{deg} / \mathrm{month}$, associated with sunspot impulses.

Gnevyshev, when finding the existence of secondary maxima, questioned the reality of Spoerer's law. This conclusion was refuted by Vitinsky who considered cyclic curves for separate latitude intervals (1965a) and has shown, by means of the dispersion analysis, the predominant role of the latitude factor in comparison with the phase factor (1965b). Tschegoleva (1965) examined cyclic curves using separate latitude zones of the sunspot number index, supporting Spoerer's law, although the latitude drift turned out different in distinct cycles and hemispheres. Ramantan and Natarayan (1965) have constructed diagrams for different longitude intervals, in which there are no violations of Spoerer's law and the 'active latitude' is found, 

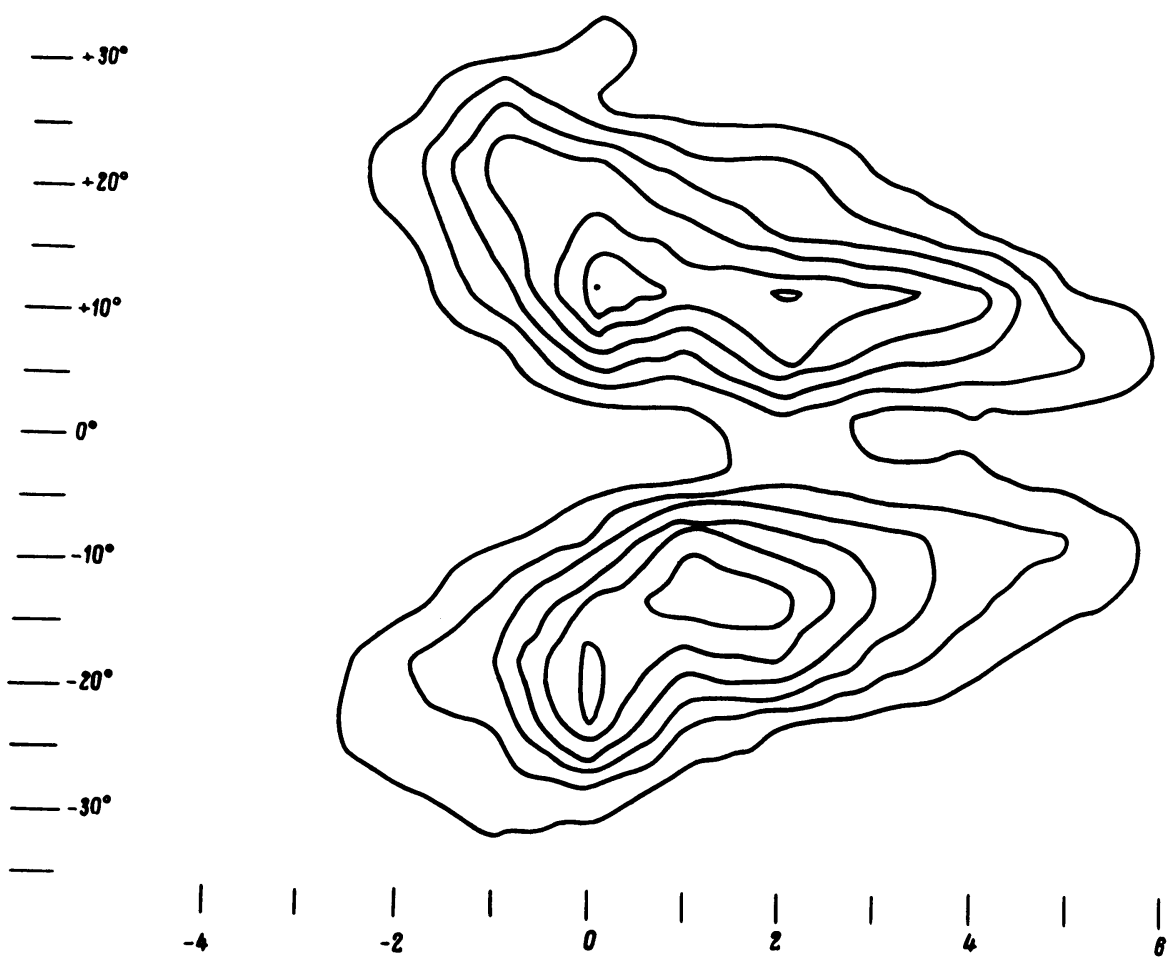

Fig. 19. The latitude-time diagram of the total sunspot area index $P$.

invariable at different cycle phases. In order to construct an average diagram for 7 cycles Kuklin (1971a) used the same data on the total-area index as Antalova and Gnevyshev (1965) utilized to disprove Spoerer's law. The obtained diagram visually demonstrates the existence of a latitude drift with many peaks. Besides, the secondary Becker zone is revealed on it with poleward directed drift (1959) the reality of which was disputed by Kopecký (1962).

All dynamics of total sunspot-area latitude distribution reflecting the important group distribution of the $E$ and $F$ classes (Kopecký and Künzel, 1962), may be revealed by solving the reverse problem of the dynamic model (Kuklin, 1971d) in application to the diagram obtained by Kuklin. The distribution of the source function which corresponds predominantly to either birth or decay of large groups, shows that the cycle 'works energetically' no farther than the second year after the maximum. Approximately, in this time, according to Vitinsky and Tschegoleva (1971), the sunspot formation zone slipping down to the equator, ceases. The meridional circulation velocity field is divided into two zones: a zone of drift to the equator below $\sim 25^{\circ}$, and a zone of drift to the pole above $\sim 25^{\circ}$. This picture also agrees with the result by Becker and with the well-known pattern of the latitude drift, according to Tuominen (1942), with a difference in this case, with the behavior of sunspot formation centers. The velocities are of order $10^{2}-10^{3} \mathrm{~cm} \mathrm{~s}^{-1}$, the estimations of the diffusion coefficient are of $10^{12} \mathrm{~cm}^{2} \mathrm{~s}^{-1}$. 


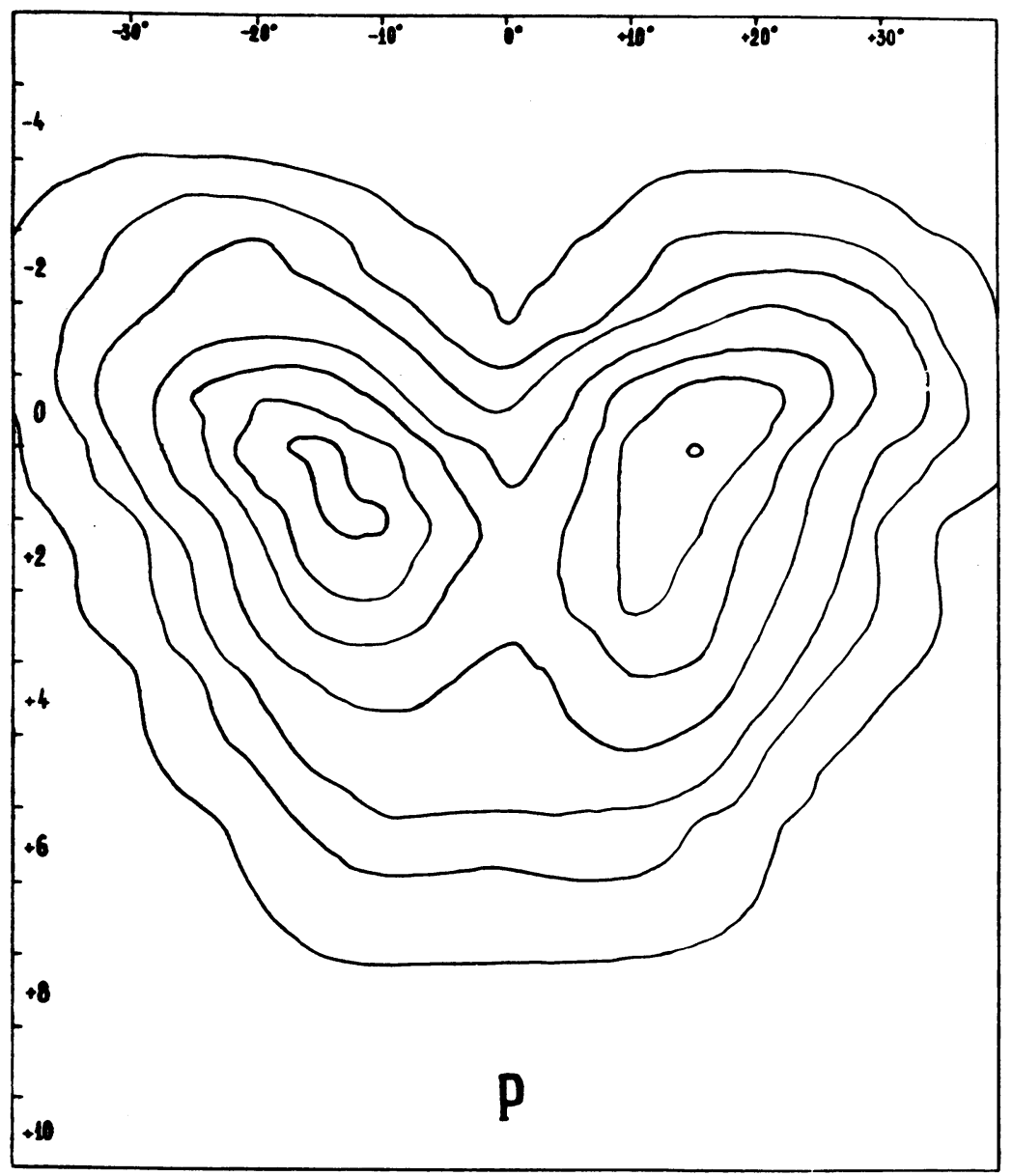

Fig. 20. The smoothed $-t$ diagram of the $P$ index.

The sunspot formation zone width on the diagram increases towards high latitudes, during cycles with high activity level (Mursalimova, 1957; Schmidt, 1962). Morozov et al. (1973) have found that within the cycle the displacements of the high latitude border occur synchronously in both latitudes. According to Gleissberg (1968), the zonal activity at different latitudes lasts from three years at high latitudes to a practically continuous one at low latitudes, the cycle height having no influence on this. In each latitude interval, the activity (and the cycle) begins with the appearance of small groups which are followed by large groups after 1-2 yr (Szymansky, 1970). The activity decay takes place in the reverse order.

One should note that the classic 'latitude-time' diagram reflects mainly the properties of the behavior of the frequency index. Kopecký showed that the diagram loses its customary regular appearance and acquires a very fluctuating structure (1962) as we consider indices in greater degree presenting the phenomena importance. The index of conditional average sunspot area, proportional to $T_{0}^{2}$, turns out to 


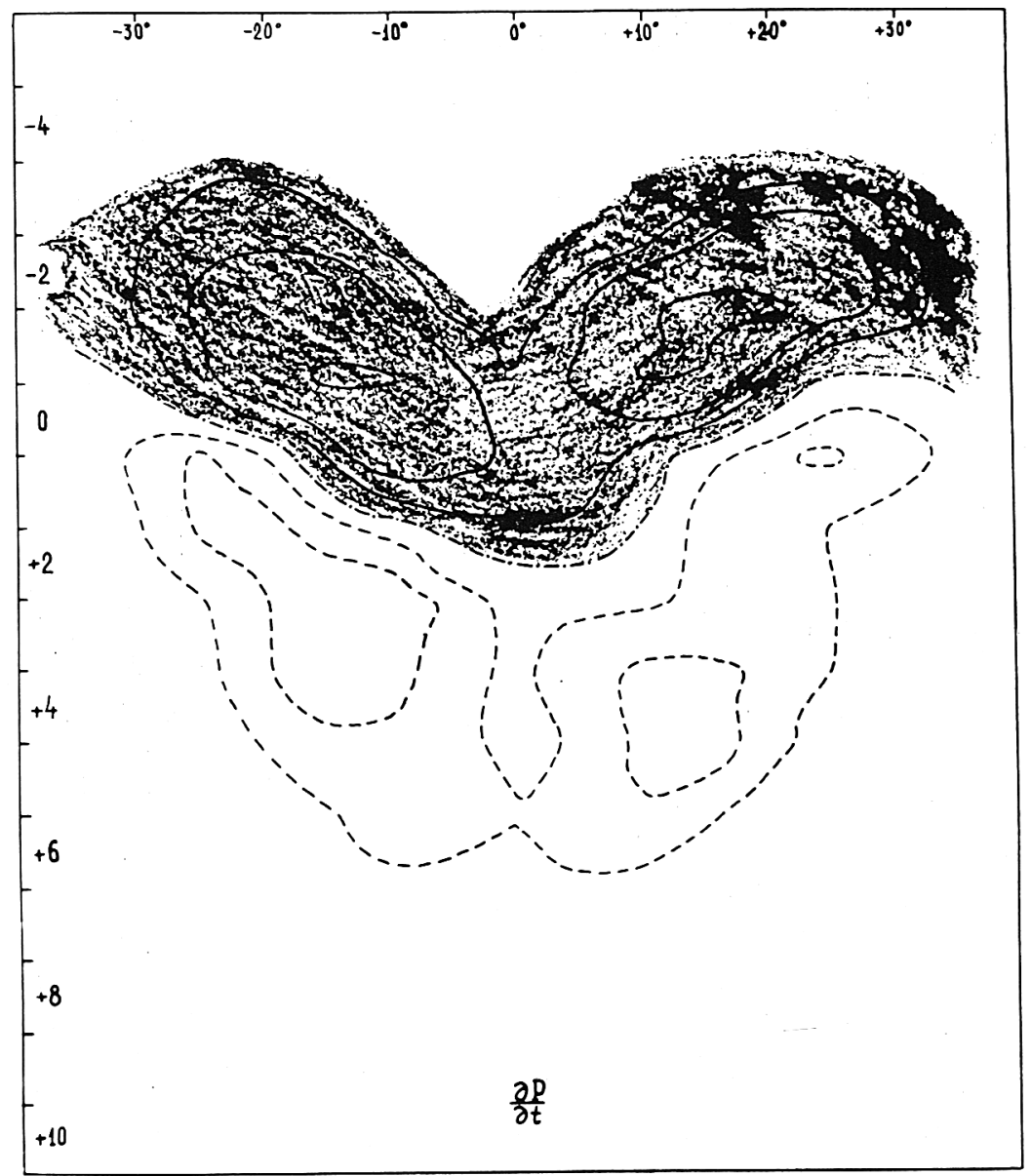

Fig. 21. The distribution of $\mathrm{d} P / \mathrm{d} t$ (positive values are shaded).

be indifferent to both the phase factor and the latitude factor (Vitinsky and Kopecký, 1968). However, on the average, the mean latitude of sunspot groups decreases with the growth of their importance (Kashirin, 1962; Ringnes, 1968).

The 'latitude-time' diagram for flares was studied by Křivsky and Knoška (1967, 1968). The equatorial drift of the zone of the highest solar activity is revealed markedly, which may be disturbed by short displacements in the opposite direction. On the diagram, fluctuations ('jerks') are present, which in the 18 cycle were located more symmetrically with respect to the equator and synchronously than in the following one.

Certain information on the magnetic field geometry of active regions may be given by investigations of the flare position with respect to sunspots. The positions of flares in latitude, according to the first results (Greatrix, 1970), were independent of the cycle phase and flare importance, but they showed a relationship with the field structure according to magnetic classifications of the groups. Detailed studies by 


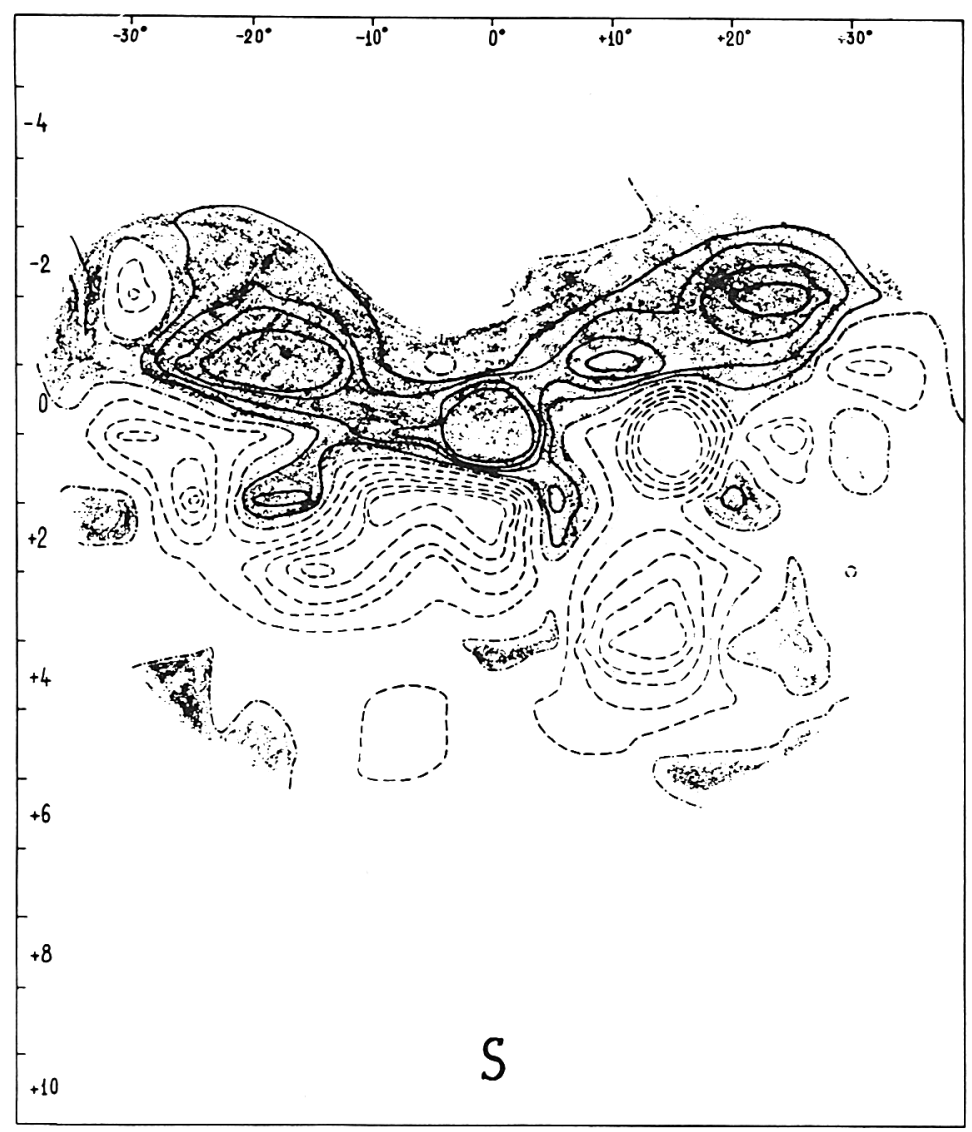

Fig. 22. The distribution of sunspot source function $S$.

Kasinsky $(1973 a, b)$ have shown that the isoline of zero displacement in latitude during the cycle drifts to the equator with a velocity of order 1 deg per month, but this motion gets broken by fluctuations. The displacement direction in latitude changes its sign at least twice a cycle. At the beginning of the cycle, the displacement is directed towards the east and the pole, and at the end - towards the west and the equator. On the diagram boundaries, the displacements are directed inside the diagram.

So, the latitude distribution of indices reflecting the frequency, varies with cycle phase according to Spoerer's law, and the importance indices exhibit a less regular pattern with pronounced concentration at low latitudes within the cycle core. The analysis of index dynamics using the 'latitude-time' diagram, reveals a number of significant details.

\subsection{ACtive LONGITUdeS}

In the longitude distribution of indices on the solar surface, inhomogeneity is expressed more significantly than in the latitude distribution, which involves the 


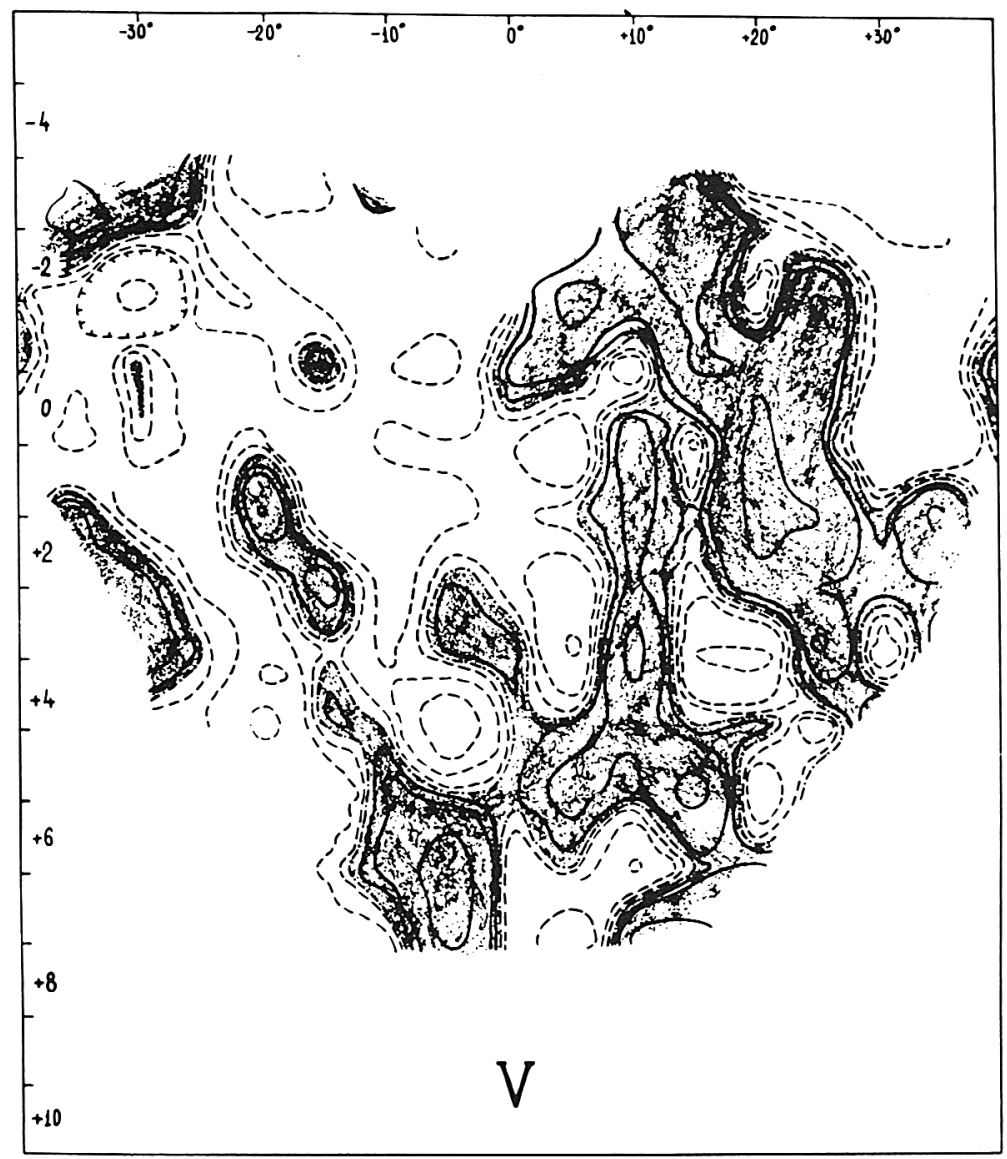

Fig. 23. The distribution of the meridional circulation velocity $V$ (isolines $0.3,1,3,10 \mathrm{~m} \mathrm{~s}^{-1}$ ).

content of the longitude distribution law of solar activity. This is expressed in terms of the existence of active longitudes, found at the beginning of the present century. The typical property of active longitudes is that they are not subjected to any effects of the differential rotation.

Active longitudes are very noticeable when data for relatively large time intervals of the order of several years or an entire cycle, are utilized. Active longitudes, as a rule, are $180^{\circ}$ apart so that they form antipodal pairs with unequal activity level. The main population of active longitudes is composed of important recurrent sunspot groups. Relatively high stability of active longitudes is observed which may exist during several cycles (Vitinsky, 1958). They may, from cycle to cycle, irregularly displace in longitude at distances less than their own size. Besides active longitudes Vitinsky introduced the concept of cycle-like longitudes (1962a). The longitude interval within which the cyclic curve differs little from the cyclic curve for the Sun, as a whole, is called the cycle-like longitude. Usually, considerations are conducted separately for both hemispheres. Generally, there are more cycle-like longitudes 


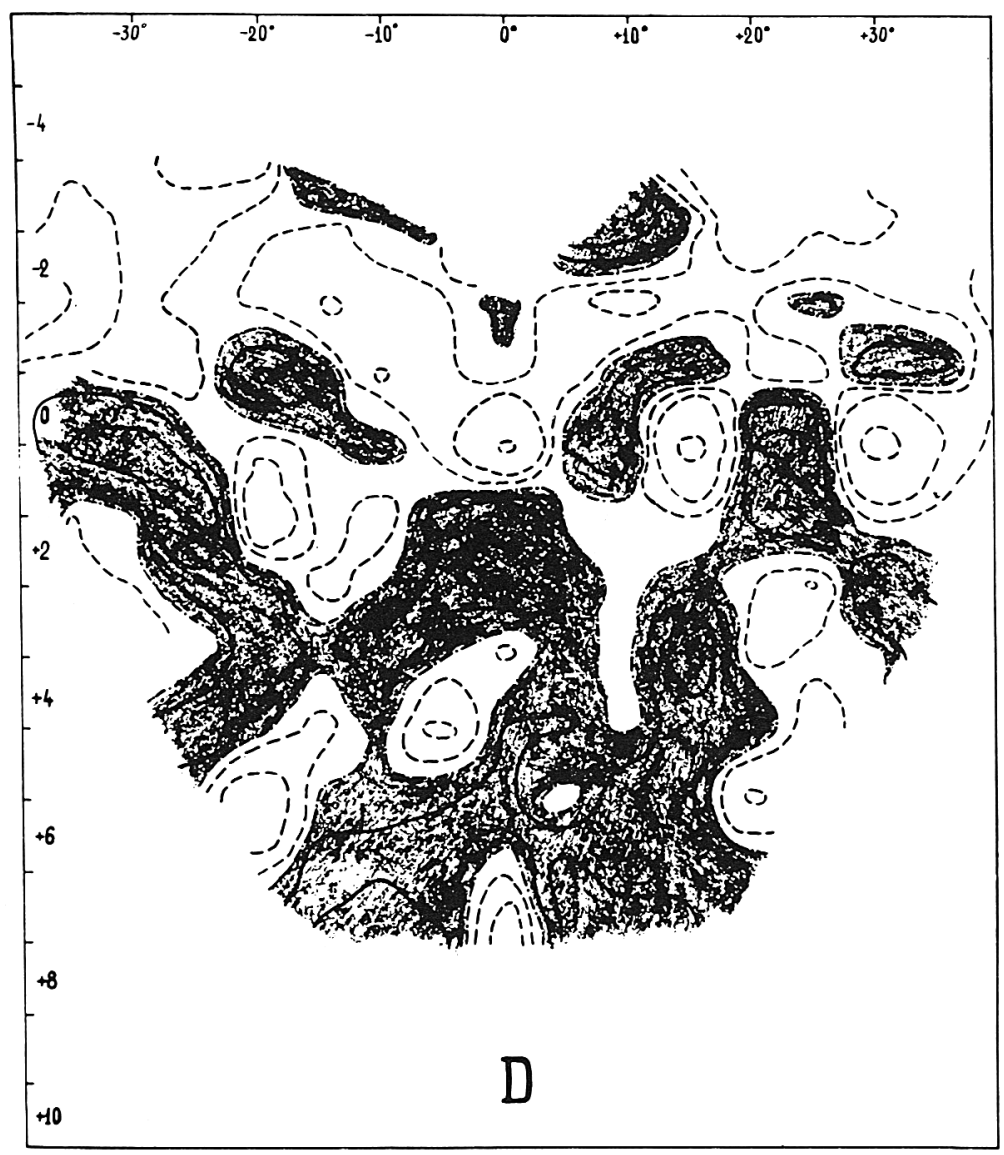

Fig. 24. The distribution of the quasi-diffusion coefficient $D$ (isolines $10^{12}$ and $3 \times 10^{12} \mathrm{~cm}^{2} \mathrm{~s}^{-1}$ ).

than active ones, and only a part of them coincides with active ones. The cycle-like nature is expressed stronger for the sunspot number than for the sunspot area. Generally, active longitudes of the sunspot number are less stable, and the number of them is less than of active longitudes of sunspot areas (Vitinsky, 1967). In 50\% of cycle-like longitudes, the solar activity level is below the average. Cycle-like longitudes remain during two or more cycles and practically determine the character of the cyclic curve for the Sun as a whole. Asynchronism in separate longitude intervals is expressed stronger than in hemispheres. Active longitudes are often characterized by an extremely fluctuated cycle evolution (Vitinsky, 1962a, b).

Asynchronism in sunspot formation was noted by Bezrukova (1963) with respect to antipodal active longitudes. Kuklin (1962) tried to distinguish active longitudes of high latitude sunspot groups. It turned out that they are not associated with the main active longitudes of the major sunspot formation zone but they may precede (1-2 yr) the secondary active longitudes of the major zone. According to Vitinsky (1968b) Spoerer's law in different longitude intervals manifests equally and the active zone width varies similarly. But if we pay attention to new sunspots in the beginning of a 
cycle, Berdichevskaya (1968) has shown that their active longitudes, from cycle to cycle, are considerably displaced, passing into the neighboring longitude quadrant in the solar rotation direction.

As was already mentioned, active longitudes may have displacements. According to Rodionov (1962) such oscillations are more typical for cycle extrema epochs and differ for active longitudes of small and large groups. Vitinsky (1963a) concluded that displacements in active longitudes increase from cycle to cycle with decreasing activity level in them. There is some evidence that in different cycles the Sun rotates differently. The problem of the existence of cyclic variations of solar rotation seems to be quite urgent (Shodo 1955, 1957).

Kozhevnikov (1970) has found active longitudes of the kinematic index, characterizing sunspot group motion velocities in latitude and longitude. They coincide with active longitudes of sunspots where, consequently, an increase in activity is caused by an increase in motions.

In the sunspot group longitude distribution periodicities were found which are different in both hemispheres (Morozov and Obashev 1969, Morozov et al. 1973). These periods vary synchronously with mean linear group dimensions but with a phase shift in different hemispheres. This effect is probably not linked directly with the existence of active longitudes but indicates structure of quite another time scale. Also Stanck (1972) has found that longitude distributions in both hemispheres are independent and are characterized by the presence of the main maxima at a distance of $90^{\circ}$ in longitude and secondary maxima at a distance of $30^{\circ}$. It is curious that he has found long-lived regions with minimal sunspot number on whose boundaries activity is increased (sunspots and prominences). Such regions are most likely to correspond to coronal holes (Kasinsky and Tomozov, 1975).

Active longitudes were also distinguished using other phenomena of solar activity. Vitinsky (1969b) has distinguished active longitudes of weak background magnetic fields which for different polarities are situated in different hemispheres. In the northern hemisphere they coincide with active longitudes of other indices. The regions of magnetic field emergence, revealed from changes in the fine structure of the chromosphere, have no predominant longitudes (Glackin, 1973). Such compact regions exist during about 10 rotations and are continuously carried away toward the east with the velocity of about $0.6 \mathrm{deg}$ per day. The coincidence of active longitudes of flares and sunspots, best expressed for important phenomena, was obtained by Vitinsky (1969a) and Maris (1972). Similar results of the coincidence of active longitudes of a great number of characteristics were obtained by Warwick (1965) for sunspots, flares, proton flares, plages, prominences, type-IV bursts, noise storms, important sunspot groups with an area of more than 1000.

The results obtained are consistent when data for large time intervals are utilized. In the case of short intervals, however, active longitude systems can be obtained with a real spectrum of values of the synodical period of solar rotation as it was shown by Wilcox and Schatten (1967) on the basis of Warwick's data. Fung et al. (1971) have discovered an interesting detail in connection with the flare longitude distribution. Active longitudes are obtained, taking into consideration all observed flares, however, if one by one the flares are sampled randomly from each active region, random distribution results. Hence, active longitudes of flares, to a considerable extent, are 
caused by flare concentration in active regions and by the existence of active longitudes in active regions.

The existence of active longitudes of calcium flocculi was shown by Godoli et al. (1966, 967). Ambrož (1973a) has examined the distribution of calcium flocculi in longitude and has tried to determine the periods, with which resulting structures rotate. He has revealed row systems rotating at different velocities due to which they form a net of intersecting straight lines on the 'longitude-time' diagram. The rows themselves exist for about 10 revolutions (active region) and form streams. The regions of crossing streams are characterized by increased activity. The whole structure has a lifetime of $20-25$ solar rotations $(1.7 \mathrm{yr})$. The only exception consists in the longitude distribution of hydrogen filaments, active longitudes of which are quite real, but they are situated within intervals between active longitudes of other indices (Vitinsky, 1973b).

Active longitudes are produced by bright formations in the corona (5303) according to the data by Sýkora (1971) and Cimakhovich (1972). According to Nesmyanovich and Khomenko (1969), active longitudes exist also for the S-component of soiar radio emission.

On the basis of the above one should expect that the activity center distribution must repeat the longitude distributions of other indices and must contain the same active longitudes. According to the results by Vitinsky (1968), at the 11-year cycle descending branch, the activity centers have both an average lifetime of 3.3 rotations and real maxima in longitude distribution. When the minimum epoch enters, the lifetime decreases 1.5 times, and distribution in longitude becomes random. Further investigations (Vitinsky, 1971b) have shown that in maximum epochs the longitude distribution of activity centers bears a random character too. However, one can reveal active longitudes during a cycle, as a whole, and they remain during two cycles. Similar studies were performed by Trellis $(1971 \mathrm{a}, \mathrm{b})$. Distribution of activity centers has a relatively stable structure, especially in longitude for zones of sunspot formations. As the mean sunspot area increases, structural stability increases (up to several cycles). Zones of increased activity are outstanding for their typical spatial distribution of the mean area index.

Therefore, for the overwhelming majority of important phenomena of solar activity, irregular longitude distribution is typical, with the formation of active longitudes existing during several cycles and not influenced by the differential rotation. These peculiarities are weaker or even are absent in distributions of indices which either reflect the frequency or describe minor formations.

\subsection{SUMMARY}

All the above confirms the concept of two classes of indices representing the frequency and importance of phenomena on the Sun. The reality of this concept is illustrated by observational data and facts. The main problem which remains is the building of a solar activity theory and that consists of a number of smaller problems. It is somewhat tiresome to tell in detail about each of these problems but the main problems must be mentioned. 
TABLE III

\begin{tabular}{|c|c|c|}
\hline Period & Frequency $11 \mathrm{yr}$ & $\begin{array}{l}\text { Importance } \\
80-90 \mathrm{yr}\end{array}$ \\
\hline $\begin{array}{l}\text { Dependence on } \\
\text { latitude }\end{array}$ & Spoerer's law & $\begin{array}{l}\text { Increase of maximal } \\
\text { latitude }\end{array}$ \\
\hline $\mathrm{N}-\mathrm{S}$ asymmetry & $?$ & $+?$ \\
\hline $\begin{array}{l}\text { Compactness of } \\
\text { active zones }\end{array}$ & $\begin{array}{l}\text { Large in latitude } \\
\text { small in longitude }\end{array}$ & Active longtitudes \\
\hline Type of rotation & Differential & Rigid \\
\hline
\end{tabular}

(1) The build up of a complete and exhaustive picture of solar activity phenomena in order to obtain a functionally adequate system model with the most important couplings.

(2) The build up of a structurally adequate model of solar activity which is the last step to a physical theory.

(3) The clearing up of the meaning of the correlations between the planet locations and the solar activity variations.

(4) The study and classification of different solar activity phenomena in respect to time and space regularities.

(5) The build up of physically consistent mathematical models of various phenomena changes.

(6) The build up of an evolution model of solar activity considering time and spatial couplings.

(7) The clearing up of sense of difference and similarity of frequency and importance indices, phenomena, concepts.

\section{References}

Afanas'ev, A. N.: 1961, Soln. Dan., No. 5, 62.

Alfvén, H.: 1945a, Monthly Notices Roy. Astron. Soc. 105, 3.

Alfvén, H.: 1945b, Monthly Notices, Roy. Astron. Soc. 105, 382.

Ambrož, P.: 1971, Solar Phys. 19, 480.

Ambrož, P.: 1973a, Bull. Astron. Inst. Czech. 24, 80.

Ambrož, P.: 1973b, Bull. Astron. Inst. Czech. 24, 130.

Anderson, C. N.: 1939, Bell. Syst. Tech. J. 18, 292.

Anderson, P. N.: 1954, J. Geophys. Res. 59, 455.

Antalova, A.: 1971, Bull. Astron. Inst. Czech. 22, 352.

Antalova, A. and Gnevyshev, M. N.: 1965, Astron. Zh. 42, 253.

Antonucci, E.: 1974, Solar Phys. 34, 471.

Basu, D. and Das Gupta, M. K.: 1966, Ind. J. Phys. $40,117$.

Bell, B.: 1960, Smithson. Contrib. Astrophys. 5, 17.

Becker, U.: 1959, Z. Astrophys. 48, 88.

Berdichevskaya, V. S.: 1967, Astron. Zh. 44, 358.

Berdichevskaya, V. S.: 1968, Astron. Zh. 45, 459.

Bezrukova, A. Ya.: 1958, Izv. Gl. Astron. Obs. Pulkovo, No. 159.

Bezrukova, A. Ya.: 1963, Izv. Gl. Astron. Obs. Pulkovo 23, 57.

Bigg, E. K.: 1967, Astron. J. 72, 463.

Blizard, J. B.: 1965, Astron. J. 70, 667.

Bonov, A. D.: 1957, Soln. Dan., No. 3.

Bonov, A. D.: 1958, Bull. Vses. Astron. Geodez. Ob., No. 21. 
Bonov, A. D.: 1961, Soln. Dan., No. 3, 56.

Bonov, A. D.: 1964, Soln. Dan., No. 3, 67.

Bonov, A. D.: 1966, Godishnik Sof. Univ. Fiz. Fak. 59, 75.

Bonov, A. D.: 1972a, Izv. Sekc. Astron. Bulg. Akad. Nauk 5, 33.

Bonov, A. D.: 1972b, Izv. Sekc. Astron, Bulg. Akad, Nauk 5, 41.

Bonov, A. D.: 1973, Izv. Sekc. Astron. Bulg. Akad. Nauk 16, 15.

Cheng Byao and In Chung-lin: 1965, Acta Astron. Sin. 13, 89.

Chertoprud, V. E.: 1966, Astron. Zh. 43, 390.

Chertoprud, V. E. and Kotov, V. A.: 1965, Astron. Tsirk., No. 318, 1.

Chirkov, N. P.: 1971, Soln. Dan., No. 11, 75.

Chistyakov, V. F.: 1959a, Soln. Dan., No. 2.

Chistyakov, V. F.: 1959b, Bull. Vses. Astron. Geodez. Ob., No. 25.

Chistyakov, V. F.: 1961, Soln. Dan., No. 7, 78.

Chistyakov, V. F.: 1963, Soln. Dan., No. 6, 65.

Chistyakov, V. F.: 1965, Izv. Gl. Astron. Obs. Pulkovo, 24, 60.

Chistyakov, V. F.: 1966, Soln. Dan., No. 8, 83.

Chistyakov, V. F.: 1968, Solnech. Aktivnost', Moskva, No. 3, 140.

Chistyakov, V. F.: 1973, Ciklicheskaya Deyatel'nost' Solnca, Vladivostok.

Cimakhovich, N. P.: 1972, Appar. Metody Obrab. Radiosatr. Nabl., Riga, p. 105.

Cohen, T. J. and Limtz, P. R.: 1974, Nature 250, 398.

Cole, T. W.: 1973, Solar Phys. 30, 103.

Covington, A. E.: 1974, J. Roy. Astron. Soc. Can. 68, 35.

Currie, R. G.: 1973, Astrophys. Space Sci. $20,509$.

Danvillier, A.: 1970, Compt. Acad. Sci. 270, B1119.

Danvillier, A.: 1973, Bull. Rend. Sci. Acad. Roy. Belg. 59, 917.

Dezsö, L. and Gerlei, O.: 1964a, Publ. Debrecen Heliophys. Observ. 1, 3.

Dezsö, L. and Gerlei, O.: 1964b, Publ. Debrecen Heliophys. Observ. 1, 35.

Dezsö, L. and Gerlei, O.: 1965, Publ. CSAV, Astron. Ustav, No. 51, 41.

Dingle, L. A. et al:: 1973, Solar Phys. 31, 243.

Djurkovič, P.: 1956, Soln. Dan., No. 6.

Dmitrieva, M. G.: 1965, Soln. Dan., No. 4.

Dmitrieva, M. G. et. al.: 1971, Issled. Geom., Aeron., Fiz. Solnca, Irkutsk, vyp. 2, 180.

Dodson, H. W. and Hedeman, R. E.: 1972, in E. R. Dyer (General Editor), Solar-Terrestrial Phys. 1970,

Dordrecht, Part 1, p. 151.

Dolginov, A. Z. et. al.: 1972, Astron. Vestn. 6, 195.

Drozdov, O. A.: 1950, Tr. Gl. Geofiz. Obs. 19(81), 102.

Eigenson, M. S.: 1963, Solnce, pogoda, klimat, Gidrometeoizdat.

Eigenson, M. S. and Mandykina, T. L.: 1962a, Cirk. Astron. Observ. Lvov Univ., No. 37-38, 80.

Eigenson, M. S. and Mandykina, T. L.: 1962b, Cirk. Astron. Observ. Lvov Univ., No. 37-38, 82.

Eigenson, M. S. and Mandykina, T. L.: 1962c, Cirk. Astron. Observ. Lvov Univ., No. 37-38, 91.

Ferris, G. A. Y.: 1969, J. Brit. Astron. Assoc. 79, 385.

Fokker, A. D.: 1963, Bull. Astron. Inst. Neth. $17,84$.

Fredga, K.: 1965, Stellar and Solar Magnetic Fields, Amsterdam, p. 310.

Fung, P. C. W. et. al.: 1971, Solar Phys. 18, 90.

Gabrovski, I. et al.: 1967, Izv. Sekc. Astron. Bulg. Akad. Nauk 2, 139.

Gabrovski, I. et. al: 1968, Geomagnetizm i Aerononsyia 8, 929.

Giovanelli, G.: 1964, Observatory 84, 57.

Glackin, D. L.: 1973, Publ. Astron. Soc. Pacific 85, 241.

Gleissberg, W.: 1955, Naturwissenschaften 42, 410.

Gleissberg, W.: 1958, Z. Astrophys. 46, 219.

Gleissberg, W.: 1966, J. Brit. Astron. Assoc. 76, 265.

Gleissberg, W.: 1968, Solar Phys. 4, 93.

Gnevyshev, M. N.: 1963, Astron. Zh. 40, 401.

Gnevyshev, M. N.: 1966, Usp. Fiz. Nauk 90, 291.

Gnevyshev, M. N.: 1967, Solar Phys. 1, 107.

Gnevyshev, M. N. and Antalova, A.: 1965, Publ. CSAV, Astron. Ustav, No. 51, 47.

Gnevyshev, M. N. and Krivsky, L.: 1966, Astron. Zh. 43, 385.

Gnevyshev, M. N. and Ol', A. I.: 1948, Astron. Zh. 38, 18.

Godoli, G. and Mazzucconi, F.: 1967, Astrophys. J. 147, 1131.

Godoli, G. et al.: 1966, Ann. Geophys. 19, 395.

Godoli, G. et al.: 1967, Ann. Geophys. 20, 265. 
Golubtsov, V. V.: 1965, Soln. Dan., No. 6, 70.

Granova, V. D. et al.: 1972, Astron. Tsirk., No. 703, 1.

Greatrix, G. R.: 1970, Astron. Astrophys. 5, 171.

Gudzenko, L. I. et al.: 1965, Astron. Tsirk., No. 342, 1.

Gudzenko, L. I. and Chertoprud, V. E.: 1962, Astron. Zh. 39, 758.

Gudzenko, L. I. and Chertoprud, V. E.: 1966, Astron. Zh. 43, 113.

Hakura, Y.: 1974, Solar Phys. 39, 493.

Howard, R. F.: 1965, Stellar and Solar Magnetic Fields, Amsterdam, p. 132.

Henkel, R.: 1972, Solar Phys. 25, 498.

Jakimiec, M.: 1969, Acta Astron. (Polska) 19, 59.

Jose, P. D.: 1965, Astron. J. 70, 193.

Kandaurova, K. A.: 1971, Soln. Dan., No. 5, 107.

Kasinsky, V. V.: 1973a, Soln. Dan., No. 2, 78.

Kasinsky, V. V.: 1973b, Soln. Dan., No. 7, 77.

Kasinsky, V. V. and Tomozov, V. M.: 1975, Soln. Dan., No. 2, 84.

Kashirin, G. F.: 1962, Cirk. Astron. Observ. Lvov Univ., No. 37-38, 96.

Kolomeets, E. V. et al.: 1974, Geomagnetizm i Aeronomyia 14, 728.

Kolomiets, A. R.: 1969, Astron. Tsirk., No. 509, 7.

Kopecký, M.: 1958a, Publ. CSAV Astron. Ustav, No. 42.

Kopecký, M.: 1958b, Bull. Astron. Inst. Czech. 9, 34.

Kopecký, M.: 1962, Bull. Astron. Inst. Czech. 13, 63.

Kopecký, M.: 1964, Bull. Astron. Inst. Czech. 15, 178.

Kopecký, M.: 1967, Adv. Astron. Astrophys., vol. 5, Academic Press, N.Y.-London, p. 189.

Kopecký, M.: 1972, Bull. Astron. Inst. Czech. 23, 107.

Kopecký, M.: 1974, Bull. Astron. Inst. Czech. 25, 271.

Kopecký, M.: 1975, Bull. Astron. Inst. Czech. (in press).

Kopecký, M. and Kuklin, G. V.: 1969, Bull. Astron. Inst. Czech. $20,22$.

Kopecký, M. and Kuklin, G. V.: 1971, Issled. Geomagn., Aeron., Fiz. Solnca, Irkutsk, vyp. 2, 167.

Kopecký, M. and Künzel, H.: 1962, Astron. Nachr. 286, 193.

Kotov, V. A. et al.: 1965a, Astron. Tsirk., No. 331.

Kotov, V. A. et. al: 1965b, Astron. Tsirk., No. 333, 6.

Kozelov, V. P.: 1972, Geofiz. Issled. v Zone pol. Siyan., Apatity, 128.

Kozelov, V. P.: 1975, Subburi $i$ vozm. v. magnitosf., Leningrad, 274.

Kozelov, V. P. and Mingaleva, G. I.: 1975, Subburi $i$ vizm. v magnitosf., Leningrad, 264.

Kozhevnikov, N. I.: 1970, Soob. Gos. Astron. Inst. Shternb., No. 162, 16.

Kozhevnikov, N. I.: 1973, Astron. Tsirk., No. 802, 3.

Kreplin, R. W.: 1970, Ann. Geophys. 26, 567.

Křivský, L. and Knoška, S.: 1967, Bull. Astron. Inst. Czech. 18, 325.

Křivský, L. and Knoška, S.: 1968, Bull. Astron. Inst. Czech. 19, 365.

Křivský, L. and Krüger, A.: 1966, Bull. Astron. Inst. Czech. 17, 243.

Kuklin, G. V.: 1962, Soln. Dan., No. 1, 72.

Kuklin, G. V.: 1971a, Soln. Dan., No. 2, 75.

Kuklin, G. V.: 1971b, Soln. Dan., No. 6, 89.

Kuklin, G. V.: 1973, Soln. Dan., No. 2, 53.

Kuklin, G. V.: 1971c, IAU Symp. 43, 737.

Kuleshova, K. F.: 1962, Astron. Zh. 39, 272.

Levitsky, L. S.: 1967a, Izv. Krymsk. Astrofiz. Observ. 37, 137.

Levitsky, L. S.: 1967b, Izv. Krymsk. Astrofiz. Observ. 37, 158.

Liese, R.: 1971, Hinweise auf Zusammenhänge zwischen den Planeten und den Sonnenfleckenperioden,

Techn. Univ. Hannover.

Link, F.: 1963, Bull. Astron. Inst. Czech. 14, 226.

Loewe, F.: 1973, Gerlands Beitr. Geophys. 82, 25.

Macris, C. J.: 1960. Mem. Nat. Obs. Athenes, ser. I, 7.

Makarenko, N. G.: 1973, Tr. Astrofiz. Inst. Akad. Nauk KazSSR 23, 92.

Maksimov, I. V.: 1952, Dokl. Akad. Nauk. SSSR 42, 1149.

Maksimov, I. V. and Smirnov, N. P. 1967, Soln. Dan., No. 10, 104.

Maris, G.: 1972, Stud. Si Cerc. Astron. 17, 71.

Minasyants, G. S. and Obashev, S. O.: 1969, Soln. Dan., No. 6, 108.

Molchanov, A. M.: 1966, Dokl. Akad. Nauk. SSSR 168, 284.

Morozov, N. N. and Obashev, S. O.: 1969, Tr. Astrofiz. Inst. Akad. Nauk KazSSR 15, 38.

Morozov, N. N. et al.: 1973, Tr. Astrofiz. Inst. Akad. Nauk KazSSR 23, 48. 
Mursalimova, G. G.: 1957, Tr. Tashk. Astron. Obs., ser. 2 5, 145.

Muzalevsky, Yu. S. and Zhukov, L. V.: 1968a, Astron. Tsirk., No. 488, 7.

Muzalevsky, Yu. S. and Zhukov, L. V.: 1968b, Soln. Dan., No. 12, 77.

Nelson, J. H.: 1963, The Effect of Disturbances of Solar Origin on Communications, Pergamon Press, p. 293.

Nemeth, T.: 1966, Pure Appl. Geophys. 63, 205.

Nesmyanovich, A. T. and Khomenko, Yu. A.: 1969, Soln. Dan., No. 12, 98.

Newcomb, T.: 1901, Astrophys. J. 13, 1.

Ol', A. I.: 1960, Astron. Zh. 37, 222.

Ol', A. I.: 1972, Soln. Dan., No. 12, 105.

Pathak, P. N.: 1972, Solar Phys. 25, 489.

Pavelyev, S. V. and Pavelyeva, Z. S.: 1965, Tr. Gl. Geofiz. Obs., vyp. 181, 92.

Phadke, M. S. and Wu, S. M.: 1974, J. Amer. Statist. Assoc. 69, 325.

Predtechensky, P. P. and Gurevich, B. S.: 1948, Tr. Gl. Geofiz. Obs. 8(70), 33.

Prokudina, V. S.: 1973a, Astron. Tsirk., No. 804, 3.

Prokudina, V. S.: 1973b, Soob. Gos. Astron. Inst. Shternb., No. 181, 11.

Ramanathan, A. S. and Nagarajan, V.: 1965, Observatory 85, 188.

Rao, K. R.: 1973, Solar Phys. 29, 47.

Rima, A.: 1961, Geophys. Meteorol. 9, 39.

Ringnes, T. S.: 1962, Astrophys. Norv. 8, 17.

Ringnes, T. S.: 1964, Astrophys. Norv. 8, 303.

Ringnes, T. S.: 1965, Astrophys. Norv. 10, 27.

Ringnes, T. S.: 1968, Astrophys. Norv. 10, 189.

Rodionov, A. V.: 1962, Cirk. Astron. Observ. Lvov Univ., No. 37-38, 99.

Romanchuk, P. R.: 1963, Geofiz. i Astron. Inform. Bull., No. 5, 248.

Romanchuk, P. R.: 1965a, Soln. Dan., No. 5, 65.

Romanchuk, P. R.: 1965b, Soln. Dan., No. 7, 65.

Romanchuk, P. R.: 1965c, Soln. Dan., No. 8, 74.

Romanhcuk, P. R.: 1974a, Vestn. Kiev. Univ. Ser. Astron., No. 16, 17.

Romanchuk, P. R.: 1974b, Vestn. Kiev. Univ. Ser. Astron., No. 16, 28.

Romanchuk, P. R. and Sergeeva, A. N.: 1974, Astrometr. $i$ Astrofiz., vyp. 24, 107.

Rubashev, B. M.: 1949, Bull. Komis. Issled. Solnca, No. 2.

Rubashev, B. M.: 1962, Soln. Dan., No. 10, 52.

Rubashev, B. M.: 1964, Problemy solnechnoj aktivnosti, Moskva-Leningrad, Nauka.

Schmidt, L.: 1962, Bull. Astron. Ist. Czech. 13, 246.

Schove, D. J.: 1955, J. Geophys. Res. 60, 127.

Shapiro, R. and Ward, F.: 1962, J. Astrophys. Sci. 19, 506.

Sheeley, N. R. Jr.: 1964, Astrophys. J. 140, 731.

Shnitnikov, A. V.: 1951, Bull. Komis. Issled. Solnca, No. 7, 47.

Shodo, E. L.: 1955, Astron. Tsirk., No. 161, 9.

Shodo, E. L.: 1957, Astron. Tsirk. No. 178, 15.

Stuvalov, V. M.: 1970, Astron. Vestn. 4, 198.

Stanek, W.: 1972, Solar Phys. 27, 89.

Suda, T.: 1962, J. Meteorol. Soc. Japan, Ser. II, 40, 287.

Sýkora, J.: 1971, Solar Phys. 18, 72.

Szymanski, W.: 1970, Postepy Astron. 18, 305.

Szymanski, W.: 1971, Postepy Astron. 19, 153.

Szymanski, W.: 1974, Postepy Astron. 22, 45.

Takahashi, K.: 1967, J. Radio Res. Labs. 14, 237.

Takahashi, K.: 1968, Solar Phys. 4, 598.

Toman, K.: 1967, J. Geophys. Res. 72, 5570.

Trellis, M.: 1966a, Compt. Rend. Acad. Sci. AB262, B221.

Trellis, M.: 1966b, Compt. Rend. Acad. Sci. AB262, B312.

Trellis, M.: 1966c, Compt. Rend. Acad. Sci. AB262, B376.

Trellis, M.: 1971a, Compt. Rend. Acad. Sci. 272, B549.

Trellis, M.: 1971b, Compt. Rend. Acad. Sci. 272, B1026.

Trellis, M.: 1973, Compt. Rend. Acad. Sci. 277, B183.

Trotter, D. E. and Billings, D. E.: 1962, Astrophys. J. 136, 1140.

Tschegoleva, G. P.: 1965, Soln. Dan., No. 8, 70.

Tuominen, J.: 1942, Z. Astrophys. 21, 96.

Turner, H.: 1925, Monthly Notices Roy. Astron. Soc. 85, 467. 
Vasilyev, O. B.: 1970, Soln. Dan., No. 1, 92.

Vasilyev, O. B. and Vitinsky, Yu. I.: 1969, Soln. Dan., No. 9, 105.

Vasilyev, O. B. and Kandaurova, K. A.: 1968, Soln. Dan., No. 12, 85.

Vasilyev, O. B. and Kandaurova, K. A.: 1970a, Soln. Dan., No. 2, 106.

Vasilyev, O. B. and Kandaurova, K. A.: 1970b, Soln. Dan., No. 11, 109.

Vasilyev, O. B. and Rubashev, B. M.: 1972, Soln. Dan., No. 1, 95.

Vasilyeva, G. Ya et al.: 1971, Soln. Dan, No. 9, 96.

Vasilyeva, G. Ya et al.: 1972a, Soln. Dan., No. 2, 99.

Vasilyeva, G. Ya et al.: 1972b, Soln. Dan., No. 8, 106.

Vasilyeva, G. Ya et al.: 1974, Soln. Dan., No. 6, 99.

Vasilyeva, G. Ya et al.: 1975, Soln. Dan., No. 2, 76.

Vertlib, A. B. and Kuklin, G. V.: 1971a, Soln. Dan., No. 5, 76.

Vertlib, A. B. and Kuklin, G. V.: 1971b, Soln. Dan., No. 9, 79.

Vertlib, A. B. and Kuklin, G. V.: 1971c, Soln. Dan., No. 10. 86.

Vitinsky, Yu. I.: 1958, Soln. Dan., No. 3.

Vitinsky, Yu. I.: 1960, Soln. Dan., No. 9, 78.

Vitinsky, Yu. I.: 1961a, Izv. Gl. Astron. Obs. Pulkovo 22, 121.

Vitinsky, Yu. I.: 1961b, Soln. Dan., No. 11, 64.

Vitinsky, Yu. I.: 1962a, Soln. Dan., No. 2, 66.

Vitinsky, Yu. I.: 1962b, Izv. G. Astron. Obs. Pulkovo 22, 111.

Vitinsky, Yu. I.: 1963a, Soln. Dan., No. 3, 64.

Vitinsky, Yu. I.: 1963b, Soln. Dan., No. 5, 71.

Vitinsky, Yu. I.: 1965a, Soln. Dan., No. 11, 62.

Vitinsky, Yu. I.: 1965b, Soln. Dan., No. 12, 53.

Vitinsky, Yu. I.: 1967, Soln. Dan., No. 6, 80.

Vitinsky, Yu. I.: 1968a, Soln. Dan., No. 2, 90.

Vitinsky, Yu. I.: 1968b, Soln. Dan., No. 3, 100.

Vitinsky, Yu. I.: 1968c, Soln. Dan., No. $9,90$.

Vitinsky, Yu. I.: 1968d, Izv. Gl. Astron. Obs. Pulkovo, No. 184, 66.

Vitinsky, Yu. I.: 1969a, Solar Phys. 7, 210.

Vitinsky, Yu. I.: 1969b, Soln. Dan., No. 4, 88.

Vitinsky, Yu. I.: 1969c, Soln. Dan., No. 10, 107.

Vitinsky, Yu. I.: 1971a, Izv. Gl. Astron. Obs. Pulkovo, No. 186, 20.

Vitinsky, Yu. I.: 1971b, Izv. Gl. Astron. Obs. Pulkovo, No. 189-190, 10.

Vitinsky, Yu. I.: 1972, Soln. Dan., No. 5, 84.

Vitinsky, Yu. I.: 1973a, Soln. Dan., No. 6, 82.

Vitinsky, Yu. I.: 1973b, Soln. Dan., No. 9, 80.

Vitinsky, Yu. I.: 1973c, Ciklichnost' i prognozy solnechnoj aktivnosti, Leningrad.

Vitinsky, Yu. I. and Ikhsanov, R. N.: 1970, Soln. Dan., No. 8, 103.

Vitinsky, Yu. I. and Kopecký, M.: 1968, Izv. Gl. Astron. Obs. Pulkovo, No. 184, 73.

Vitinsky, Yu. I. and Rubashev, B. M.: 1974, Soln. Dan., No. 1, 84.

Vitinsky, Yu. I. and Tschegoleva, G. P.: 1971, Soln. Dan., No. 3, 74.

Vlasov, V. A. et al.: 1974, Kratkie soob. po fiz., No. 12, 9.

Waldmeier, M.: 1935, Astron. Mitt. Zürich, No. 133.

Waldmeier, M.: 1957, Z. Astrophys. 43, 149.

Waldmeier, M.: 1966, Astron. Mitt. Zürich, No. 274.

Waldmeier, M.: 1973, Solar Phys. 28, 389.

Warwick, C. S.: 1965, Astrophys. J. 141, 500.

Wilcox, J. M. and Colburn, D. S.: 1969, J. Geophys. Res. 74, 2388.

Wilcox, J. M. and Schatten, K. H.: 1967, Astrophys. J. 147, 364.

Wood, R. M. and Wood, K. D.: 1965, Nature, 208, 129.

Xanthakis, J.: 1960, Prakt. tis Akad. Athen. 35, 352.

Xanthakis, J.: 1962a, Mem. Soc. Astron. Ital. 33, 291.

Xanthakis, J.: 1962b, Ann. Astrophys. 25, 342.

Xanthakis, J.: 1966, Bull. Astron. Inst. Czech. 17, 215.

Xanthakis, J.: 1969, Solar Phys. 10, 168.

Xanthakis, J.: 1970, Compt. Rend. Acad. Sci. 271, B1009.

Zabza, M.: 1962, Acta Astron. (Polska) 12, 210.

Zabza, M.: 1964, Prace Wrocl. towarz. nauk, B, No. 112, 57.

Zhukov, L. V. and Muzalevsky, Yu. S.: 1969, Soln. Dan., No. 8, 88.

Zhukova, L. N. and Mitrofanova, L. A.: 1973, Soln. Dan., No. 6, 65. 


\section{DISCUSSION}

Krause: Estimations of the energy of the solar magnetic fields are strongly depending on the model you consider. For example, if you interpolate the $1 \mathrm{G}$ surface field as a dipole-like-field which penetrates the whole Sun you will get quite a different value compared with a model where the a.c.-character is taken into account and which penetrates therefore only a surface layer. The same is true for the differential rotation. There are suggestions that the Sun under the convection zone rotates much faster. So my question is, on what models have you based your estimations of the magnetic energy and the differential rotation?

Kuklin: The first estimation is the value of rotation energy, not only of differential rotation energy. The second one is the value of convection motion energy and the differential rotation energy is of the same order if using well known formula for differential rotation.

Stix: How deep, then, do the sunspots extend?

Kuklin: My estimations are limited within a layer of $20000 \mathrm{~km}$ thickness but I took into account the fact that small sunspots have smaller depth and used the sunspot distribution in respect to sunspot area and assumed $H \sim L / 3$. 